\title{
Energy-aware and Carbon-efficient VM Placement Optimization in Cloud Datacenters Using Evolutionary Computing Methods
}

\section{Tahereh Abbasi-khazaei}

Islamic Azad University Science and Research Branch

Mohammad Hossein Rezvani ( $\nabla$ mhossein.rezvani@gmail.com )

Qazvin Islamic Azad University https://orcid.org/0000-0002-0292-7008

\section{Research Article}

Keywords: cloud computing, virtual machine placement, energy consumption, renewable energy, carbon footprint, cost optimization

Posted Date: January 12th, 2022

DOI: https://doi.org/10.21203/rs.3.rs-1233833/v1

License: (c) (i) This work is licensed under a Creative Commons Attribution 4.0 International License.

Read Full License 


\title{
Energy-aware and Carbon-efficient VM Placement Optimization in Cloud Datacenters using Evolutionary Computing Methods
}

\author{
Tahereh Abbasi-khazaei \\ Department of Computer Engineering \\ Science and Research Branch, Islamic Azad University \\ Tehran, Iran \\ tahereh.abbasi@srbiau.ac.ir
}

Tel. Number: +98-9019429214

\author{
Mohammad Hossein Rezvani* \\ Faculty of Computer and Information Technology Engineering \\ Qazvin Branch, Islamic Azad University \\ Qazvin, Iran \\ rezvani@qiau.ac.ir
}

Tel. Number: +98-9126870533

\section{*Corresponding Author: Mohammad Hossein Rezvani, rezvani@qiau.ac.ir}

ORCID: 0000-0002-0292-7008

\begin{abstract}
One of the most important concerns of cloud service providers is balancing renewable and fossil energy consumption. On the other hand, the policy of organizations and governments is to reduce energy consumption and greenhouse gas emissions in cloud data centers. Recently, a lot of research has been conducted to optimize the Virtual Machine (VM) placement on physical machines to minimize energy consumption. Many previous studies have not considered the deadline and scheduling of IoT tasks. Therefore, the previous modelings are mainly not well-suited to the IoT environments where requests are time-constraint. Unfortunately, both the subproblems of energy consumption minimization and scheduling fall into the category of NP-hard issues. In this study, we propose a multi-objective VM placement to joint minimizing energy costs and scheduling. After presenting a modified memetic algorithm, we compare its performance with baseline methods as well as stateof-the-art ones. The simulation results on the CloudSim platform show that the proposed method can reduce energy costs, carbon footprints, SLA violations, and the total response time of IoT requests.
\end{abstract}

Keywords: cloud computing, virtual machine placement, energy consumption, renewable energy, carbon footprint, cost optimization

\section{1- Introduction}

A big challenge with cloud data centers is high power consumption. In general, the cost of electricity and the cost of maintaining a cloud data center are two of the major costs imposed. The cost of electricity is a more important factor. Studies show that an idle server consumes about 50\%-70\% of its maximum power. Also, according to Amazon estimates, the energy cost of a data center accounts for $40 \%$ of its total Operational Expenditures (OPEX) [1]. The rate of carbon generated in a cloud data center depends on the type of energy source it consumes. It varies for different energy sources such as oil, coal, and renewable energy. Thus, even a small reduction in energy consumption leads to significant cost savings and a carbon footprint. Much of this 
problem can be alleviated through the dynamic placement of Virtual Machines (VMs). For this purpose, the real-world parameters affecting the Virtual Machine Placement (VMP) must be considered in the modeling. Optimizing energy consumption directly affects carbon footprint and carbon taxation, which in turn can reduce the cost of cloud service providers.

In the past, a lot of research has been done on the VMP to physical machines. The main challenge in this regard is to perform the VMP in such a way that minimizes energy consumption and at the same time satisfies users' Service Level Agreement (SLA) and Quality of Experience (QoE). Some researchers have used classical mathematics methods [2, 3, 4, 5]. The VMP falls into the category of bin-packing problems, which itself is an NP-hard issue [6, 7]. Some researchers have used heuristic methods to reduce resource allocation time $[1,8,9,10]$. Another way to reduce convergence time on NP-hard problems is to use metaheuristic methods $[6,7,8,11,12,13,14,15]$.

Recently, the emergence of the Internet of Things (IoT) has generated tremendous volumes of big data. Many of these data come from delay-sensitive applications. Some of the most important applications are agriculture [16], vehicular traffic management [17, 18], e-health [19], and so on. Each IoT request is assigned to a VM to be executed. Then each VM must in turn be mapped to a suitable physical server using VMP approaches. Here, it is important to pay attention to the deadline of tasks. Unfortunately, many previous studies have merely focused on optimizing green energy consumption and have by no means considered task scheduling. Therefore, previous modelings are mainly not suitable for IoT environments where requests are time-constraint. Scheduling optimization, like energy efficiency, falls into the category of NP-hard problems.

In this study, we propose a multi-objective VMP algorithm for joint minimization of energy costs and scheduling. After presenting a modified memetic algorithm, we compare its performance with baseline methods such as the Genetic Algorithm (GA) as well as state-of-the-art methods. Roughly speaking, we seek to answer the question of how to optimize the VMP concerning the SLA and the QoE of users, while considering both energy and scheduling constraints. Some of our most important assumptions are:

- Each IoT task has a deadline.

- We have information about changes in the outdoor temperature of the servers in a certain period.

- Solar energy is considered a major type of renewable energy.

- Datacenters are considered geographically distributed.

- Energy costs vary for data centers that are geographically dispersed.

The most important innovations of this research are as follows:

- We formulate the two sub-problem of energy cost optimization and IoT task scheduling optimization. Then, we solve them jointly with the deterministic weighted sum method, which is one of the well-known methods of nonlinear optimization. To the best of our knowledge, no research has been conducted on the joint optimization of green energy and task scheduling.

- Because the problem is NP-hard, we solve it on a large scale using a modified MA method. Then, we evaluate the performance of the proposed method against the baseline method such as GA as well as state-of-the-art methods.

The remainder of this paper is organized as follows: Section 2 reviews the most important studies on energy-efficient VMP; Section 3 explains the problem formulation; Section 4 describes the proposed method; 
Section 5 evaluates the results of simulating the proposed method on the CloudSim simulator [20]; Finally, Section 6 concludes the study and provides suggestions for future research.

\section{2- Related Work}

So far, there has been a great deal of literature on cloud service energy cost minimization. Before reading this section, interested readers are highly recommended to refer to [2] for a comprehensive survey regarding the architecture. After studying previous researches, we divided them into three categories including, exact (classic mathematical), heuristic, and meta-heuristic. For each of these categories, we present the purpose, limitations, methodology, and evaluation criteria in Tables 1 and 2.

Some researchers address the issue of VMP. To solve the carbon dioxide problem, $\mathrm{Xu}$ and Buyya [4] proposed a strategy for managing the greenhouse gas and renewable energy effects for several data centers in California, Virginia, and Dublin that are located in different time zones. Their results show that the proposed algorithm could reduce carbon emissions by nearly $40 \%$ compared to state-of-the-art data centers while guaranteeing an average response time to user requests. Other related researches can be found in [1]. New research using classical mathematics and heuristic methods is shown in Table1.

Table 1- Researches done with exact (classic mathematical) and heuristic methods

\begin{tabular}{|c|c|c|c|c|c|}
\hline Ref. & Year & Objective & Constraints & Evaluation Criteria & Method \\
\hline$[2]$ & 2019 & $\begin{array}{l}\text { Energy consumption } \\
\text { estimates }\end{array}$ & $\begin{array}{l}\text { - Heterogeneity of VM in } \\
\text { size (CPU and RAM) } \\
\text { - Execute all VMs during the } \\
\text { period } \\
\text { - Uniform distribution of } \\
\text { VMs } \\
\text { - Number of computer nodes } \\
\text { for all architectures }\end{array}$ & $\begin{array}{l}\text { Total energy } \\
\text { consumption } \\
\text { Dynamic energy } \\
\text { consumption }\end{array}$ & exact \\
\hline$[3]$ & 2019 & $\begin{array}{l}\text { Reducing energy } \\
\text { consumption }\end{array}$ & $\begin{array}{l}\text { - VM migration limit } \\
\text { - Select the host where the } \\
\text { VM migrated } \\
\text { - Refrain from } \\
\text { overloading/underloading } \\
\text { the new host } \\
\text { Host Mips. }\end{array}$ & $\begin{array}{l}\text { Energy consumption } \\
\text { - VM migrations } \\
\text { The standard deviation } \\
\text { of the execution time } \\
\text { The average execution } \\
\text { time }\end{array}$ & exact \\
\hline
\end{tabular}




\begin{tabular}{|c|c|c|c|c|c|}
\hline Ref. & Year & Objective & Constraints & Evaluation Criteria & Method \\
\hline [4] & 2019 & $\begin{array}{l}\text { Reduce total } \\
\text { greenhouse gas } \\
\text { emissions } \\
\text { Reduce the } \\
\text { response time }\end{array}$ & $\begin{array}{l}\text { Do not exceed the capacity } \\
\text { of the VM } \\
\text { The utilization of the VM to } \\
\text { execute requests should not } \\
\text { exceed the capacity of the } \\
\text { physical machine. }\end{array}$ & $\begin{array}{l}\text { - Comparison of brown } \\
\text { energy consumption } \\
\text { (such as fossil energy) } \\
\text { for several algorithms } \\
\text { - Comparison of emitted } \\
\text { carbon in each } \\
\text { algorithm } \\
\text { - Average response time } \\
\text { - Total cost }\end{array}$ & exact \\
\hline$[10]$ & 2018 & $\begin{array}{c}\text { Reducing the amount } \\
\text { of energy required } \\
\text { by the power grid }\end{array}$ & $\begin{array}{l}\text { - PUE } \\
\text { - Datacenter battery capacity } \\
\text { - The capacity of solar panels }\end{array}$ & $\begin{array}{l}\text { - Energy price } \\
\text { - Hourly production of } \\
\text { renewable energy } \\
\text { - Energy consumption } \\
\text { - Energy cost } \\
\text { - Electric energy } \\
\text { demand }\end{array}$ & heuristic \\
\hline
\end{tabular}

Another group of studies has used meta-heuristic methods. Gao et al. [21] developed an algorithm inspired by Ant Colony Optimization (ACO). The goal is to obtain a set of non-dominant solutions (Pareto Optimal set) that simultaneously minimizes the waste of total resources and energy consumption. Parvizi et al. [6] solved the VMP problem using a Non-dominated Sorting Genetic Algorithm (NSGA-III) to minimize total resource loss and minimize energy consumption and also the number of active physical machines. For this purpose, a multiobjective optimization problem was designed and after introducing a nonlinear convex optimization solution, it is solved by the NSGA-III method. Also, in [14], the optimization of VMP in heterogeneous multi-cloud systems by considering the peak time of demand and the geographical location of allocated resources was presented, which aims to reduce energy costs. The authors also presented a dynamic energy model, designed for physical machines and cloud communication components. In some researches such as $[8,22,23]$ the GA has been used. In previous researches, besides the above-mentioned methods, other methods such as the artificial bee algorithm [7], the Cuckoo algorithm [9], etc. have been used.

Iwendi et al. [11] developed a method to minimize the energy consumption of sensors in the IoT network by combining the Whale Optimization Algorithm (WOA) with the Simulated Annealing (SA). They tried to select the most suitable Cluster Heads $(\mathrm{CH})$ in the IoT network by considering several criteria such as the number of nodes, load, temperature, and residual energy. Maddikunta et al. [24] conducted a study with the same objectives, but this time combining the WOA and a Butterfly Flame Optimization (MFO) algorithm. RM et al. [12] proposed a new architecture to minimize energy consumption in the IoT. Their proposed method first tries to minimize energy consumption by clustering different IoT networks using a wind-driven optimization algorithm. Next, using the Firefly algorithm, an optimized CH is selected for each cluster. Their method reduces data traffic compared to other non-clustered state-of-the-art designs. The $\mathrm{CH}$ selection is a complex issue in 
almost all types of networks. For example, it is common for wireless sensor networks to use evolutionary methods to select $\mathrm{CHs}$ to minimize energy consumption. To see how to model such systems, you can refer to [25].

Optimization methods are also widely used in other fields of engineering. Vashishtha et al. [26] proposed a deep-learning scheme to identify bucket defects in the Pelton wheel. Initially, the raw vibration signal is transmitted through a time-varying filter. The parameters of the filter are optimized by the Amended Grey Wolf Optimization (AGWO). They use the Convolutional Neural Network (CNN) model to train the dataset. Another example is the minimization of passband, stopband, and transition band errors for the design of a two-channel quadrature mirror filter bank [27]. Interested readers can refer to [28, 29, 30] to see more engineering applications of such optimization methods.

Table 2- Researches done with meta-heuristic methods

\begin{tabular}{|c|c|c|c|c|}
\hline Ref. & Year & Objective & Constraints & Evaluation Criteria \\
\hline$[15]$ & 2018 & $\begin{array}{l}\text { maximizing the performance } \\
\text { of physical machines } \\
\text { minimizing energy } \\
\text { consumption }\end{array}$ & $\begin{array}{l}\text { maximum number of VMs running on } \\
\text { a physical machine } \\
\text { Energy consumption and performance } \\
\text { limitation }\end{array}$ & $\begin{array}{l}\text { - energy consumption } \\
\text { - utilization }\end{array}$ \\
\hline [14] & 2019 & $\begin{array}{l}\text { Reducing energy consumption } \\
\text { Reducing the VM migration } \\
\text { overhead }\end{array}$ & $\begin{array}{l}\text { - Physical machine capacity constraint } \\
\text { - Cloud input/output bandwidth } \\
\text { capacity } \\
\text { - Each VM can only be placed on one } \\
\text { physical machine } \\
\text { - Migration time window }\end{array}$ & $\begin{array}{l}\text { - Energy cost } \\
\text { - Energy consumption } \\
\text { - The average number of } \\
\text { VM migrations per time } \\
\text { slot } \\
\text { a VM migration overhead } \\
\text { - Temporal complexity }\end{array}$ \\
\hline$[6]$ & 2020 & $\begin{array}{l}\text { Minimizing resource loss } \\
\text { minimizing energy } \\
\text { consumption } \\
\text { minimizing the use of active } \\
\text { physical machines }\end{array}$ & $\begin{array}{l}\text { Each VM must be placed on only one } \\
\text { physical machine } \\
\text { - memory constraint } \\
\text { - Processor constraint }\end{array}$ & $\begin{array}{l}\text { - Average runtime } \\
\text { - Pareto optimal points } \\
\text { - Average waste of resources } \\
\text { - Average energy } \\
\text { consumption } \\
\text { The average number of } \\
\text { active physical machines }\end{array}$ \\
\hline [11] & 2021 & $\begin{array}{l}\text { minimizing the energy } \\
\text { consumption of sensors }\end{array}$ & $\begin{array}{l}\text { - distance between the normal node and } \\
\text { the cluster head }\end{array}$ & $\begin{array}{l}\text { - the number of nodes } \\
\text { - load } \\
\text { - temperature } \\
\text { - residual energy }\end{array}$ \\
\hline
\end{tabular}

In summary, the most important differences between this study and the above research are as follows: 
- Some studies $[11,12,24,25]$, have tried to balance the load while minimizing response time. Although load balancing may reduce response time, it does not necessarily reduce energy consumption. In this research, our focus is not on load balancing and the selection of $\mathrm{CHs}$.

- Many previous studies $[1,6,8,9]$ focus exclusively on minimizing energy consumption within the data center and pay no attention to the outside. In this study, we consider both energy consumption during offloading from the end device to the base station and renewable energy consumption within the data center.

- None of the above research considers satisfying the time constraint for IoT tasks. This makes modeling inconsistent with real-world requirements, especially for delay-sensitive tasks. In this study, for the first time, we consider the joint minimization of brown energy consumption and scheduling. Although optimizing these two conflicting goals may slightly increase energy consumption, it improves the overall acceptance ratio of users. The results of our simulation confirm this claim.

\section{3- System Model}

In this section, we formulate the energy consumption optimization sub-problem and the scheduling optimization problem, respectively. Then, by combining them, we present a joint optimization problem.

\section{3-1- Main Parameters}

In this study, the total cost of the data center, $c^{\text {total }}$, consists of two components: the cost of energy consumption of the system, $c^{\text {energy }}$, and the cost of carbon dioxide produced, $c^{F P}$. Also, we assume that three types of energy sources can be used to supply power to each data center. First, we try to use green or renewable energy (for example, solar energy) if available. This will reduce the cost incurred by carbon dioxide. If renewable energy is not available, off-site grid energy can be used. Finally, if these two energies are not available, brown energies, such as diesel generators, can be used in the cloud data center in an emergency. Several geographically distributed data centers are interconnected through the same network infrastructure. The notations used in this research are shown in Table 3.

\section{- The Datacenter Energy Efficiency}

We use the Power Usage Effectiveness (PUE) metric to report the performance of a data center. Introduced by Green Grid (a non-profit organization of IT professionals) in 2007. This metric has become the most common metric for reporting "energy efficiency". Organizations such as the Uptime Institute track and report the average PUE of data centers using numerous surveys [31]. Conceptually, PUE is the power used by IT devices in a data center divided by the total amount of power consumed [32]. The higher the PUE, the more energy is wasted in data centers to maintain servers [1]. Accordingly, companies such as Google and Facebook have greatly reduced their PUE in recent years by focusing on the utilization and design of custom hardware. Thus, a value of 1 for PUE is the most ideal for a data center and indicates that $100 \%$ of the energy provided by each piece of equipment is used by it. The coefficients of this equation have already been calculated by Khosravi et al. [1] based on the results in [33] as follows: 


$$
\operatorname{PUE}\left(u_{d c_{j}}(t), h_{d c_{j}}(t)\right) ; 1+\frac{0.2+0.01 u_{d c_{j}}(t)+0.01 u_{d c_{j}}(t) h_{d c_{j}}(t)}{u_{d c_{j}}(t)}
$$

Interested readers can refer to [1] to see how the coefficients of the above equation are calculated.

\section{- Overhead Power}

We denote the overhead energy to run the virtual machine $v_{i}$ by $E_{v_{i}}^{\text {overhead }}$. Today, server cooling systems are one of the most important elements that have a remarkable effect on overhead energy costs in the system.

\section{- Server Energy}

A previous study by Gao et al. [21] has shown that there is a linear relationship between the power of a server $h_{l}$ and its CPU utilization, $u_{h_{l}}(t)$ :

$$
P_{h_{l}}(u, t)=P_{h_{l}}^{i d l e}+\left(P_{h_{l}}^{\text {peak }}-P_{h_{l}}^{\text {idle }}\right) u_{h_{l}}(t)
$$

, where $P_{h_{l}}^{\text {idle }}$ and $P_{h_{l}}^{\text {peak }}$ are the average energy values of the server $h_{l}$ at idle and peak loads, respectively.

\section{- Energy Cost}

Let denote the cost of brown, renewable, and off-site energies consumed to run a virtual machine $v_{i}$ on the server by $c_{\text {energy }}^{\text {brown }}, c_{\text {energy }}^{\text {green }}$, and $c_{\text {energy }}^{\text {offsite }}$, respectively. Here energy consumption is calculated in cents per kilowatthour (centKWh). Although the cost of renewable energy, $c_{\text {energy }}^{\text {green }}$, is negligible, the cost of the other two energies is significant. Depending on the geographical distribution of data centers, the amount of these costs depends on governmental policies in different countries.

\section{- Density/Taxation of Carbon Dioxide}

The density of carbon dioxide produced by data centers can vary depending on the type of energy source and location. In this research, three types of energy sources have been used and the carbon density for renewable energy has been considered zero. The rate of carbon dioxide produced is calculated in tons per Megawatt-hour (TonMWh). Due to environmental concerns, especially global warming, some countries calculate the tax on carbon dioxide produced in dollars per tonne (Dollars Tonne) to implement environmental sustainability. In this study, since we consider the density of carbon produced from a renewable energy source to be zero, the relevant tax cost is also zero.

\section{- Temperature}

The next major variable that has a large effect on the PUE is the temperature outside the data center. Let $h_{d c_{j}}(t)$ denote the outside temperature of a data center $d c_{j}$ at time $t$. The higher the outdoor temperature of a server, 
the higher the energy consumption of the cooling system. This, in turn, will lead to a significant increase in the PUE.

Table 3. Notations used in this study

\begin{tabular}{|c|c|}
\hline Symbol & Symbol Description \\
\hline$D$ & Total number of data centers \\
\hline $\mathrm{D}=\left\{d_{1}, d_{2}, \ldots, d_{D}\right\}$ & Data centers set \\
\hline$H$ & The total number of physical servers in a data center \\
\hline $\mathrm{H}=\left\{h_{1}, h_{2}, \ldots, h_{H}\right\}$ & Set of physical servers in a data center \\
\hline $\mathrm{E}$ & The set of energy resources \\
\hline$V$ & Total number of virtual machine requests \\
\hline $\mathrm{V}=\left\{v_{1}, v_{2}, \ldots, v_{V}\right\}$ & A set of virtual machine applications \\
\hline $\mathbf{X}_{V \times D}$ & $\begin{array}{l}\text { A matrix with binary elements to show the virtual machine mapping status to the } \\
\text { data center }\end{array}$ \\
\hline$x_{v_{i}}^{d_{j}}$ & $\begin{array}{l}\text { Each element of the matrix } \mathbf{X}_{V \times D} \text { to show the mapping of a virtual machine } v_{i} \\
\text { to a data center } d_{j}\end{array}$ \\
\hline $\mathbf{Y}_{1 \times 3}=\left(y_{v_{i}}^{b r}, y_{v_{i}}^{g r}, y_{v_{i}}^{o f f}\right)$ & $\begin{array}{l}\text { A row vector that indicates the virtual machine } v_{i} \text { is mapped to the } \\
\text { brown/green/off-site power supply network }\end{array}$ \\
\hline$y_{v_{i}}^{b r}$ & $\begin{array}{l}\text { The } v_{i} \text { element of the row vector } \mathbf{Y}_{1 \times 3} \text { that indicates the virtual machine } v_{i} \text { is } \\
\text { mapped to the brown power supply network }\end{array}$ \\
\hline$y_{v_{i}}^{g r}$ & $\begin{array}{l}\text { The } v_{i} \text { element of the row vector } \mathbf{Y}_{1 \times 3} \text { that indicates the virtual machine } v_{i} \text { is } \\
\text { mapped to the green power supply network }\end{array}$ \\
\hline$y_{v_{i}}^{o f f}$ & $\begin{array}{l}\text { The } v_{i} \text { element of the row vector } \mathbf{Y}_{1 \times 3} \text { that indicates the virtual machine } v_{i} \text { is } \\
\text { mapped to the off-site power supply network }\end{array}$ \\
\hline $\mathbf{Z}_{V \times H}$ & $\begin{array}{l}\text { Matrix with binary elements to show the virtual machine mapping status to the } \\
\text { server }\end{array}$ \\
\hline$z_{v_{i}}^{h_{j}}$ & $\begin{array}{l}\text { Each element of the matrix } \mathbf{Z}_{V \times H} \text { that shows the mapping status of the virtual } \\
\text { machine } v_{i} \text { to the server } h_{j}\end{array}$ \\
\hline$t_{v_{i}}^{h l d}$ & maintenance time of the virtual machine $v_{i}$ \\
\hline$c^{T}$ & The total cost of energy and carbon \\
\hline
\end{tabular}




\begin{tabular}{|c|c|}
\hline$c_{v_{i}}^{d_{j}}$ & Cost of running virtual Machine $v_{i}$ on the data center $d_{j}$ \\
\hline$c^{E}$ & Energy cost \\
\hline$c^{F P}$ & The cost of carbon footprint \\
\hline$c_{v_{i}}^{S}$ & The cost of server energy to run a virtual machine $v_{i}$ \\
\hline$c_{v_{i}}^{O}$ & Overhead energy cost to run a virtual machine $v_{i}$ \\
\hline$c_{v_{i}}^{E}$ & The cost of energy to run a virtual machine $v_{i}$ \\
\hline$E_{v_{i}}^{T}$ & Total energy to run a virtual machine $v_{i}$ \\
\hline$E_{v_{i}}^{S}$ & Server energy to run the virtual machine $v_{i}$ \\
\hline$E_{v_{i}}^{O}$ & Overhead energy for running a virtual machine $v_{i}$ \\
\hline$E_{S}^{b r} / E_{S}^{g r} / E_{S}^{o f f}$ & $\begin{array}{l}\text { Power consumed in the form of local brown / local green/offsite to run a virtual } \\
\text { machine on the server }\end{array}$ \\
\hline$c_{E}^{b r} / c_{E}^{g r} / c_{E}^{o f f}$ & $\begin{array}{l}\text { The cost of local brown / local green/offsite energy used to run virtual machine } \\
v_{i} \text { on the server }\end{array}$ \\
\hline$P_{h_{l}}^{\text {peak }}$ & power consumption by a physical server $h_{l}$ at peak \\
\hline$P_{h_{l}}^{i d l e}$ & power consumption by a physical server $h_{l}$ at idle state \\
\hline$u_{h_{l}}(t)$ & Utilization of a physical server $h_{l}$ at a time $t$ \\
\hline$P_{h_{l}}(u, t)$ & Power consumption of a physical server $h_{l}$ at a time $t$ with the utilization $u$ \\
\hline$P_{h_{l}}\left(u, t^{+}\right)$ & $\begin{array}{l}\text { Power consumption of a physical server } h_{l} \text { with the execution of a new virtual } \\
\text { machine and with the utilization } u\end{array}$ \\
\hline$P^{O}$ & Overhead energy \\
\hline$u_{d_{j}}(t)$ & Utilization of a data center $d_{j}$ at time $t$ \\
\hline$h_{d_{j}}(t)$ & Outside temperature of a data center $d_{j}$ at time $t$ \\
\hline$E_{v_{i}}^{O, b r} / E_{v_{i}}^{O, g r} / E_{v_{i}}^{O, o f f}$ & $\begin{array}{l}\text { Local brown/local green / offsite energy overhead used to run a virtual machine } \\
v_{i}\end{array}$ \\
\hline$c_{v_{i}}^{E, b r} / c_{v_{i}}^{E, g r} / c_{v_{i}}^{E, o f f}$ & $\begin{array}{l}\text { Cost of consuming local brown / local green/offsite energy used to run a virtual } \\
\text { machine } v_{i}\end{array}$ \\
\hline$r_{F P}^{b r} / r_{F P}^{g r} / r_{F P}^{o f f}$ & $\begin{array}{l}\text { The carbon production rate in local brown / local green/offsite energy used to } \\
\text { run a virtual machine } v_{i}\end{array}$ \\
\hline$c_{t a x}^{b r} / c_{t a x}^{g r} / c_{t a x}^{o f f}$ & Carbon tax on energy resources in the form of local brown / local green/offsite \\
\hline
\end{tabular}




\begin{tabular}{|c|c|}
\hline$c p u_{v_{i}}$ & Required processing capacity of a virtual machine $v_{i}$ \\
\hline mem $_{v_{i}}$ & The required memory capacity of a virtual machine $v_{i}$ \\
\hline$s t r_{v_{i}}$ & The required storage capacity of a virtual machine $v_{i}$ \\
\hline$c p u_{h_{l}}$ & Available processing capacity of a physical machine $h_{l}$ \\
\hline mem $_{h_{l}}$ & The available memory capacity of a physical machine $h_{l}$ \\
\hline$s t r_{h_{l}}$ & The available storage capacity of a physical machine $h_{l}$ \\
\hline$E_{d_{j}}^{T, g r}$ & The total green energy available at the data center $d_{j}$ \\
\hline$E_{d_{j}}^{T, b r}$ & The total brown energy available at the data center $d_{j}$ \\
\hline$L_{v_{i}}$ & The size of a request on a virtual machine $v_{i}$ (bytes) \\
\hline$\tau_{h_{j}}$ & The time duration of each instruction inside a physical server $h_{j}(\mathrm{sec})$ \\
\hline$\theta_{h_{j}}$ & The size of each instruction inside a physical server $h_{j}$ (byte) \\
\hline$t^{\text {trans }}$ & $\begin{array}{l}\text { The transmission delay of each byte of request from a user device to the cloud } \\
\text { datacenter (sec) }\end{array}$ \\
\hline$t_{v_{i}}^{o f f l o a d}$ & The offloading latency of a request the IoT device $i$ to the cloud datacenter (sec) \\
\hline$t_{i, j}^{p r c}$ & The processing latency of a request $v_{i}$ on a physical server $h_{j}(\mathrm{sec})$ \\
\hline$t_{v_{i}}^{\text {deadline }}$ & The deadline of an IoT request that is running on a virtual machine $v_{i}(\mathrm{sec})$ \\
\hline$t_{i, j}^{r e s p}$ & $\begin{array}{l}\text { The response time of an IoT request that is running on a virtual machine } v_{i} \text { when } \\
\text { it is placed on a physical server } h_{j}\end{array}$ \\
\hline$t_{v_{i}}^{r e s p}$ & The response time of an IoT request that is running on a virtual machine $v_{i}$ \\
\hline$t^{R}$ & The aggregate response time of all IoT requests \\
\hline
\end{tabular}

\section{3-2- Cost Optimization Sub-problem}

Here, we formulate the objective function and constraints of the energy efficiency sub-problem.

\section{- Objective Function}

Our goal is to reduce the total cost of the system, $c^{T}$, which is the summation of energy cost, $c^{E}$, and the cost of producing carbon dioxide, $c^{F P}$ :

$$
c^{T}=c^{E}+c^{F P}
$$

We write

$$
c^{T}=\sum_{v_{i} \in \mathrm{V}} \sum_{d_{j} \in \mathrm{D}} c_{v_{i}}^{d_{j}} x_{v_{i}}^{d_{j}}
$$


We denote each element of the matrix $\mathbf{X}_{V \times D}$ by $x_{v_{i}}^{d_{j}}$. If the decision variable $x_{v_{i}}^{d_{j}}$ takes 1 , it means assigning $v_{i}$ to the data center $d_{j}$. Now, we describe each of the components of the above equation:

\section{a) Energy cost}

The cost of energy, $c^{E}$, can be divided into two components. The first component, $c_{v_{i}}^{S}$, is the cost of energy consumed to execute a request by the server. The second component, $c_{v_{i}}^{O}$, is the cost of overhead energy to execute the virtual machine $v_{i}$ 's request:

$$
c^{E}=\sum_{v_{i} \in \mathrm{V}}\left(c_{v_{i}}^{S}+c_{v_{i}}^{O}\right)
$$

Note that the cost of the carbon tax is calculated separately. Given that three different types of energy sources are used in three geographically distributed regions with different financial policies, the costs incurred to execute the request on servers can have different amounts. This energy is calculated as follows:

$$
c_{v_{i}}^{S}=\sum_{\varepsilon \in\{b r, g r, o f f\}} E_{v_{i}}^{S, \varepsilon} . c^{E, \varepsilon}
$$

Also, the power consumption of the server to run a virtual machine $v_{i}$ is as follows:

$$
\begin{gathered}
E_{v_{i}}^{S, b r}=y_{v_{i}}^{b r} \cdot E_{v_{i}}^{S} \\
E_{v_{i}}^{S, g r}=y_{v_{i}}^{g r} \cdot E_{v_{i}}^{S} \\
E_{v_{i}}^{S, o f f}=y_{v_{i}}^{o f f} \cdot E_{v_{i}}^{S}
\end{gathered}
$$

Depending on which of the three energy sources $\varepsilon=\{b r, g r$, off $\}$ is used, the decision variable $y_{v_{i}}^{\varepsilon}$ takes different values from the vector $\mathrm{Y}^{\varepsilon}$. If the decision variable $y_{v_{i}}^{\varepsilon}$ has a value of 1 , it means that the corresponding energy source is allocated to the virtual machine $v_{i}$.

The energy used by the server at time $t$ to execute the request of the virtual machine $v_{i}$ is calculated from the following equation:

$$
\begin{gathered}
E_{v_{i}}^{S}=\Delta P_{h_{l}} t_{v_{i}}^{\text {hld }} \\
\Delta P_{h_{l}}=\left(P_{h_{l}}\left(u, t^{+}\right)-P_{h_{l}}(u, t)\right) \\
\Delta P_{h_{l}}=\left(P_{h_{l}}^{\text {peak }}-P_{h_{l}}^{\text {idle }}\right)\left(u_{h_{l}}\left(t^{+}\right)-u_{h_{l}}(t)\right)
\end{gathered}
$$

We now proceed to calculate the second term of Eq. (5), the overhead energy cost. Note that the overhead cost varies depending on which energy source we use: 


$$
c_{v_{i}}^{O}=\sum_{\varepsilon \in\{b r, g r, o f f\}} E_{v_{i}, \varepsilon}^{O, c^{E, \varepsilon}}
$$

We now calculate the overhead energy to run the virtual machine $v_{i}$. Here, similar to Eq. (7), we use the energy resource mapping matrix to the virtual machine to calculate the overhead energy:

$$
\begin{gathered}
E_{v_{i}}^{O, b r}=y_{v_{i}}^{b r} E_{v_{i}}^{O} \\
E_{v_{i}}^{O, g r}=y_{v_{i}}^{g r} E_{v_{i}}^{O} \\
E_{v_{i}}^{O, o f f}=y_{v_{i}}^{o f f} E_{v_{i}}^{O}
\end{gathered}
$$

To calculate the overhead energy, $E_{v_{i}}^{O}$, we will have a procedure similar to Eq. (8):

$$
E_{v_{i}}^{O}=\Delta P^{O} t_{v_{i}}^{h l d}
$$

Now to calculate Eq. (13), we need to calculate the power overhead, $P^{O}$. From the definition of PUE we can write:

$$
\begin{gathered}
\operatorname{PUE}\left(u_{d_{j}}(t), h_{d_{j}}(t)\right)=\frac{P^{T}}{P_{h_{l}}(u, t)}=\frac{P^{O}+P_{h_{l}}(u, t)}{P_{h_{l}}(u, t)} \\
P^{O}=P_{h_{l}}(u, t) .\left(\operatorname{PUE}\left(u_{d_{j}}(t), h_{d_{j}}(t)\right)-1\right)
\end{gathered}
$$

By replacing Eq. (14) in Eq. (13), we obtain

$$
\begin{aligned}
E_{v_{i}}^{O} & =\left(P_{h_{l}}\left(u, t^{+}\right)+P_{h_{l}}(u, t)\right)\left(\operatorname{PUE}\left(u_{d_{j}}(t), h_{d_{j}}(t)\right)-1\right) t_{v_{i}}^{h l d} \\
E_{v_{i}}^{O} & =\Delta P_{h_{l}}{ }_{v_{v_{i}}}^{\text {hld }}\left(\operatorname{PUE}\left(u_{d_{j}}(t), h_{d_{j}}(t)\right)-1\right)
\end{aligned}
$$

\section{b) Cost of Carbon Dioxide Production}

The cost of carbon production and the carbon tax, which was first mentioned in Eqs. (3)-(4), are formulated as follows:

$$
c^{F P}=\sum_{v_{i} \in \mathrm{V}} \sum_{\varepsilon \in\{b r, g r, o f f\}} c_{v_{i}}^{E, \varepsilon} \cdot r_{F P}^{\varepsilon} \cdot c_{\text {tax }}^{\varepsilon}
$$

Similar to what we did in Eqs. (7) and (12), we can write the following relationships using resource mapping vectors to virtual machines:

$$
\begin{aligned}
& c_{v_{i}}^{E, b r}=y_{v_{i}}^{b r} \cdot c_{v_{i}}^{E} \\
& c_{v_{i}}^{E, g r}=y_{v_{i}}^{g r} \cdot c_{v_{i}}^{E}
\end{aligned}
$$




$$
c_{v_{i}}^{E, o f f}=y_{v_{i}}^{o f f} \cdot c_{v_{i}}^{E}
$$

We have already mentioned that the tax on carbon by renewable energy sources is zero. If the other two types of energy are not available, brown energy can be used when necessary. Based on this, Eq. (16) can be rewritten as follows:

$$
c^{F P}=\sum_{v_{i} \in \mathrm{V}} c_{v_{i}}^{E, o f f} \cdot r_{F P}^{o f f} . c_{\text {tax }}^{o f f}
$$

\section{- Constraints}

The constraints to be considered for the objective function of the problem are as follows:

- Renewable energy sources have the highest priority, and if green energy is limited or unavailable, data centers may use external grid energy.

- The total capacity of the processor, RAM, and storage for virtual machines running on a server $h_{l}$ should not exceed its capacity. So we write

$$
\begin{aligned}
& \sum_{v_{i} \in \mathrm{V}} \sum_{h_{j} \in \mathrm{H}} c p u_{v_{i}} \cdot z_{v_{i}}^{h_{j}} \leq c p u_{h_{j}} \\
& \sum_{v_{i} \in \mathrm{V}} \sum_{h_{j} \in \mathrm{H}} m e m_{v_{i}} \cdot z_{v_{i}}^{h_{j}} \leq m e m_{h_{j}} \\
& \sum_{v_{i} \in \mathrm{V}} \sum_{h_{j} \in \mathrm{H}} s t r_{v_{i}} \cdot z_{v_{i}}^{h_{j}} \leq s t r_{h_{j}} .
\end{aligned}
$$

The decision variable $z_{v_{i}}^{h_{j}}$ is a binary variable of the matrix $\mathbf{Z}_{V \times H}$. If the value of this variable is equal to 1 , it means that the virtual machine $v_{i}$ is located on the server $h_{j}$; otherwise, it will have a value of 0 .

- Every virtual machine $v_{i}$ should use only one power source at a time. So,

$$
y_{v_{i}}^{b r}+y_{v_{i}}^{g r}+y_{v_{i}}^{o f f}=1, \quad \forall v_{i} \in \mathrm{V}
$$

- Each element of the virtual machine mapping matrix must be non-negative:

$$
y_{v_{i}}^{b r}, y_{v_{i}}^{g r}, y_{v_{i}}^{o f f} \geq 0, \quad \forall v_{i} \in \mathrm{V}
$$

- The total local energy consumption to run the virtual machine on the server should not exceed the total green energy capacity $E_{d_{j}}^{T, g r}$ and total brown energy $E_{d_{j}}^{T, b r}$ available to the data center. So, we write

$$
\sum_{v_{i} \in \mathrm{V}}\left(E_{v_{i}}^{S, g r}+E_{v_{i}}^{O, g r}\right) \leq E_{d_{j}}^{T, g r}
$$




$$
\sum_{v_{i} \in \mathrm{V}}\left(E_{v_{i}}^{S, b r}+E_{v_{i}}^{O, b r}\right) \leq E_{d_{j}}^{T, b r}
$$

- Each virtual machine must be placed exclusively on one server. So,

$$
\sum_{h_{j} \in \mathrm{H}} z_{v_{i}}^{h_{j}}=1, \quad \forall v_{i} \in \mathrm{V}
$$

Finally, based on the above constraints and the objective function that we defined in Eqs. (3) and (4), the optimization problem is defined as follows:

$$
\begin{aligned}
& \mathrm{P}_{1}: \min _{(X, \mathrm{Y}, \mathrm{Z})} f_{1}: c^{T} \\
& \text { s.t. } \quad \text { Eqs. (19)-(23) }
\end{aligned}
$$

The above sub-problem was first solved by Khosravi et al. [1] using a heuristic method. Then, Rashida et al. [14] solved it with another heuristic method. Also, Xu and Buyya [4] solved it from the perspective of energy resource management with different assumptions. Given that in VMP, there are multiple data centers and within each, there are several servers with different capacities, it falls into the bin-packing problems. Previous researchers have shown that bin-packing is an NP-hard problem [34]. When the number of requests increases, the space for problem solutions becomes very large.

The First-fit Decreasing (FFD) algorithm is one of the simplest heuristic algorithms to solve the bin-packing problem, which provides a quick but often non-optimal solution [35]. The pseudo-code of the FFD algorithm is shown in Algorithm 1. The greedy_Allocation procedure used in this algorithm is also shown in Algorithm 2. Suppose several requests for a virtual machine $v_{i}$ arrive in the cloud broker. A buffer is used to store these requests. Initially, the buffer list is sorted in descending order of priority based on several parameters. VM requests are then sorted in descending order based on the number of required processor cycles. In case this parameter is equal for the requests, the required processor speed is used. If the above parameters are the same, the requests are sorted based on the memory parameter. Finally, if all of the above parameters are equal for requests, the amount of hard drive capacity required to execute the VM request is used as the sorting parameter. After sorting the list, the first data center is searched to select appropriate hosts for VM requests. If no suitable host is found in the current data center, the next data center will be searched. In this way, the most suitable data center and the hosts inside it are selected.

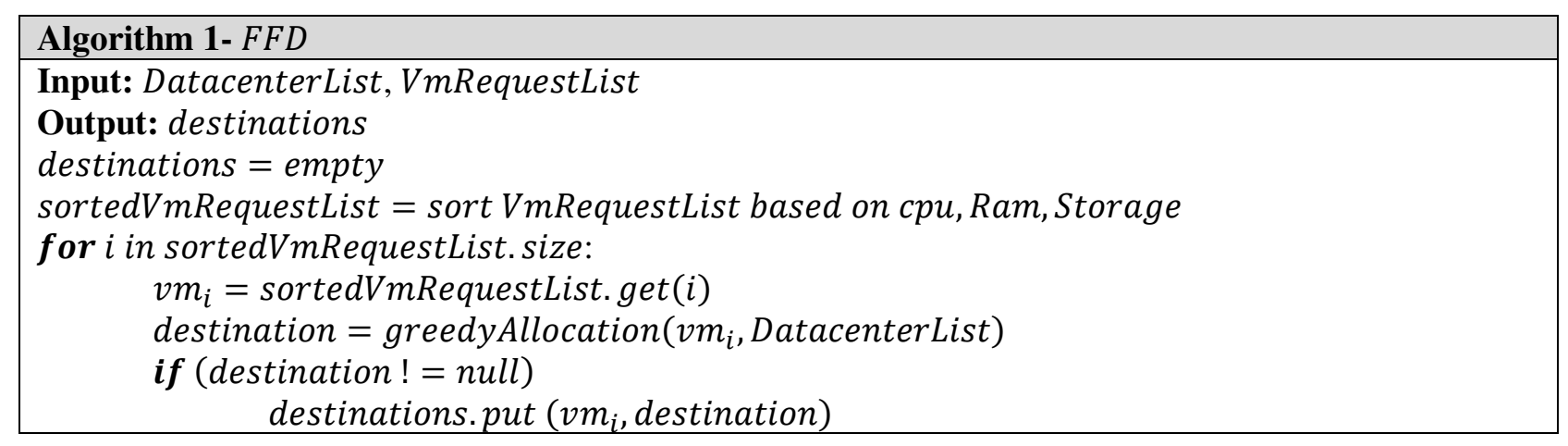




\begin{tabular}{|c|}
\hline else \\
reject $v m_{i}$ \\
return destinations \\
\hline
\end{tabular}

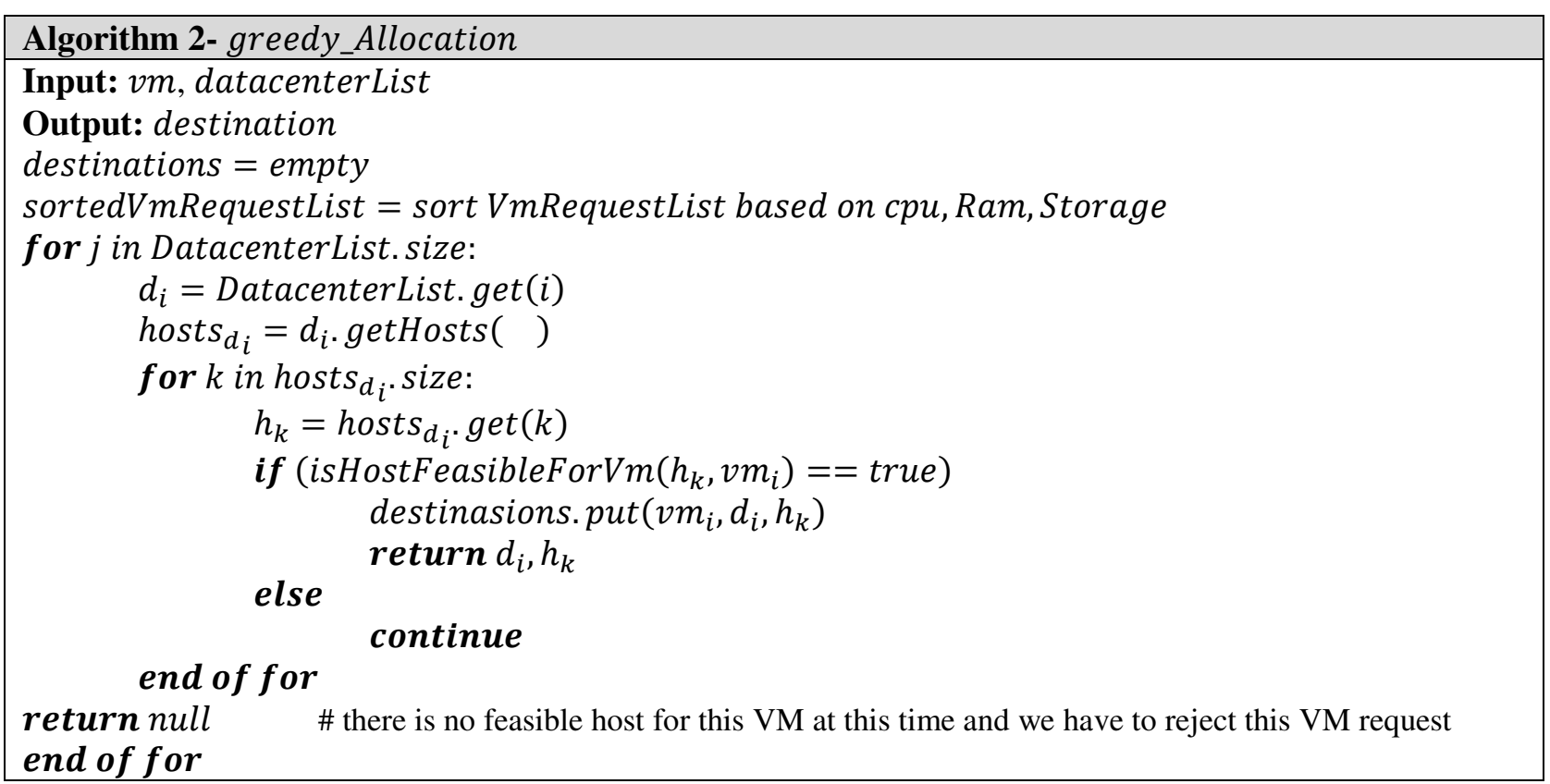

The FFD method may require a lot of execution time for longer lists. Therefore, for large-scale problems, it is better to use evolutionary algorithms.

\section{3-3- Scheduling Optimization Sub-problem}

It is important to note that each IoT request must be executed on a VM instance. Therefore, in the following, we will use the terms "IoT request" and "VM instance" interchangeably. The response time of a request $i$ sent by an IoT object consists of the sum of (a) the transmission latency to offload an object from the IoT instance $i$ to the fog instance $f, t_{v_{i}}^{\text {offload }}$, (b) the processing latency of the request $i$ inside a fog instance, $t_{i, j}^{\text {prc }}$. Total response time in the physical host $h_{j} \in \mathrm{H}$ for the request $v_{i} \in \mathrm{V}$ is obtained as follows:

$$
t_{i, j}^{\text {resp }}=t_{v_{i}}^{\text {offload }}+t_{i, j}^{\text {prc }}, \quad \forall v_{i} \in \mathrm{V} \quad \exists h_{j} \in \mathrm{H}
$$

We now proceed to calculate $t_{v_{i}}^{\text {offload }}$ in Eq. (26). We write:

$$
t_{v_{i}}^{\text {offload }}=L_{v_{i}} \cdot t^{\text {trans }}
$$

, where $t^{\text {trans }}$ denotes the transmission delay of each byte of request from an IoT device to the physical server.

Note that here we have ignored the propagation delay. We now obtain $t_{i, j}^{p r c}$ in Eq. (26): 


$$
t_{i, j}^{p r c}=L_{v_{i}} \cdot \frac{\tau_{h_{j}}}{\theta_{h_{j}}}
$$

, where, $\tau_{h_{j}}$ is the time duration of each instruction inside a physical server (in seconds). Also, $\theta_{h_{j}}$ is the size of each instruction on a physical server (in bytes). Therefore, $\tau_{h_{j}} / \theta_{h_{j}}$ denotes the time duration of each byte of the IoT request to be executed on the physical server. Multiplying this value by the request size, $L_{v_{i}}$, gives us the processing time, $t_{i, j}^{p r c}$, of the IoT request $v_{i}$ on the physical server $h_{j}$.

As mentioned earlier, a value of 1 for the binary decision variable $z_{v_{i}}^{h_{j}}$ indicates that a virtual machine $v_{i} \in \mathrm{V}$ is placed on a physical host $h_{j} \in \mathrm{H}$; otherwise, it is zero. According to Eq. (26), the response time function for an IoT request running on a virtual machine $v_{i} \in \mathrm{V}$ can be defined as follows:

$$
t_{v_{i}}^{\text {resp }}=\sum_{h_{j} \in \mathrm{H}} t_{i, j}^{\text {resp }} \cdot z_{v_{i}}^{h_{j}}, \quad \forall v_{i} \in \mathrm{V}
$$

, and the aggregate response time of all requests is as follows:

$$
t^{R}=\sum_{v_{i} \in \mathrm{V}} t_{v_{i}}^{r e s p}
$$

The response time of each IoT request running on a virtual machine $v_{i} \in \mathrm{V}$ should not exceed its deadline. That is,

$$
t_{v_{i}}^{\text {resp }} \leq t_{v_{i}}^{\text {deadline }}, \forall v_{i} \in \mathrm{V}
$$

The sub-problem of scheduling optimization is defined as follows:

$$
\mathrm{P}_{2}: \min _{(\mathrm{Z})} t^{R}
$$

s.t. $\quad$ Eqs. (19), (23), and (31)

The above relation states that our goal is to minimize the overall response time of the system, provided that the deadline of each task is met.

\section{3-4- Joint Cost and Scheduling Optimization Problem}

As mentioned earlier, our goal is to find the best candidates from the physical host nodes to place any IoT requests to. The placement operation must be performed in such a way that leads to a trade-off between response time and energy cost in the system.

Finally, the optimization problem is formulated as follows:

$$
\mathrm{P}_{3}: \min _{(X, \mathrm{Y}, \mathrm{Z})}\left\{f_{1}: c^{T} \& f_{2}: t^{R}\right\}
$$

s.t. $\quad$ Eqs. (19)-(23), and (31) 
The above multi-objective problem can be converted to a single-objective form as follows [36]:

$$
\begin{gathered}
\mathrm{P}_{4}: \min _{(X, \mathrm{Y}, \mathrm{Z})} F=w_{1} \cdot \operatorname{Ln}\left(f_{1}\right)+w_{2} \cdot \operatorname{Ln}\left(f_{2}\right) \\
\text { s.t. } \quad \text { Eqs. (19)-(23), and (31) } \\
\qquad w_{1}+w_{2}=1 \\
0 \leq w_{1} \leq 1 \\
0 \leq w_{2} \leq 1
\end{gathered}
$$

The use of the convex function of the logarithm in Eq. (36) guarantees the existence of the optimal solution [6]. Unfortunately, as mentioned earlier, both sub-problems of task scheduling and energy efficiency fall into the category of NP problems. Therefore, deterministic methods fail to obtain a solution. In the next section, we solve this problem using a metaheuristic method.

In this research, for the first time, we solve the above problem using two meta-heuristic methods of genetic algorithm (GA) and memetic algorithm (MA).

\section{4- Proposed Metaheurisic Methods}

We consider the method presented in [1] as the baseline method to solve the sub-problem of Eq. (24). We call this method, "Cost and Renewable Energy-Aware Dynamic PUE" or in abbreviation CRA-DP. Then, we solve the joint cost and scheduling optimization problem and propose two metaheuristic algorithms to overcome the NP-hardness and time complexity of the problem.

\section{4-1- Genetic Algorithm}

In this section, we present a genetic-based approach to the VMP problem of Eq. (24). We call this method, "Cost and Renewable Energy-Aware Dynamic PUE with Genetic Algorithm" or in abbreviation CRA-DP-GA. In this method, there is a population that consists of several chromosomes. Each chromosome is in the form of an array. The number of cells in this array is equal to the number of VMs, $N_{V}$. The value stored inside each cell is the identifier of a physical machine. Each cell of the chromosome is called a gene. Fig. 1 shows a possible solution to the problem of Eq. (24).

$\forall i \in\left\{1, \ldots, N_{D C}{ }^{*} N_{H}\right\}$
\begin{tabular}{|l|l|l|l|l|l|}
\hline$p m(i)$ & $p m(i)$ & $p m(i)$ & $\ldots$ & $p m(i)$ & $p m(i)$ \\
\hline$v m(1)$ & $v m(2)$ & $v m(3)$ & $\ldots$ & $v m\left(N_{V}-1\right)$ & $v m\left(N_{v}\right)$
\end{tabular}

Fig. 1- The structure of each chromosome in the CRA-DP-GA 


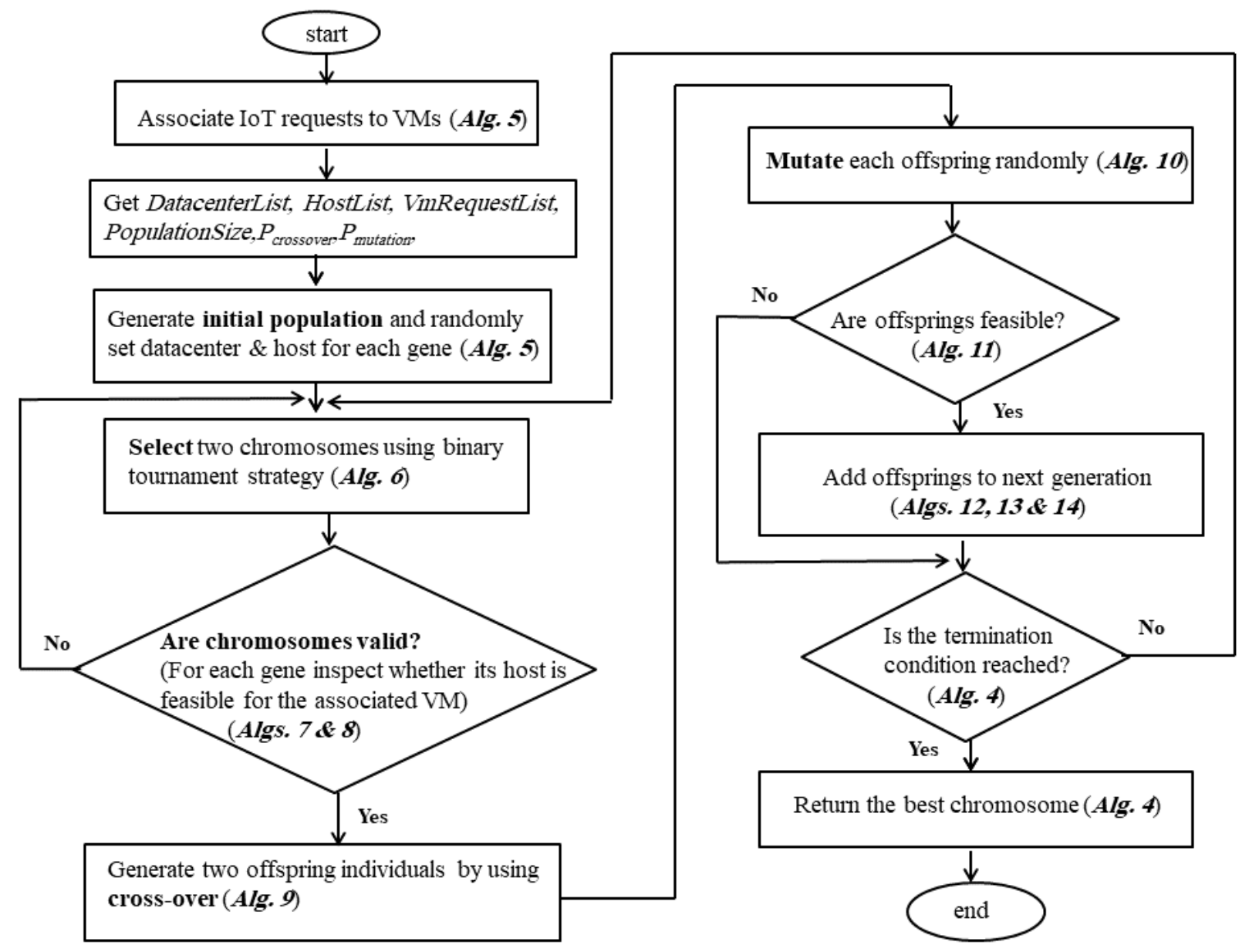

Fig. 2- Flowchart of the CRA-DP-GA

\section{4-1-1- Initial Population}

The method of selecting the initial population may have an important effect on the speed of convergence [17]. If no information is available, the random method is used [14]. Accordingly, in this study, we use a random method to generate the initial population. A pseudo-code for the initial population is provided in Algorithm 5.

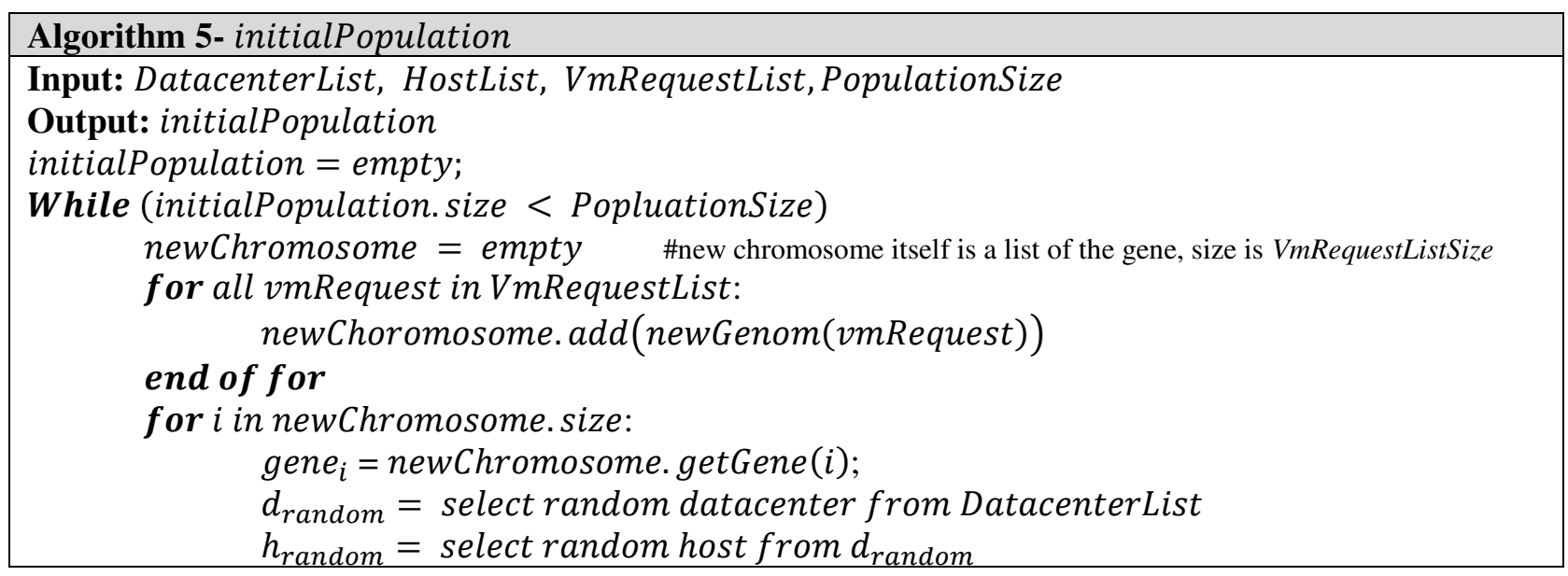




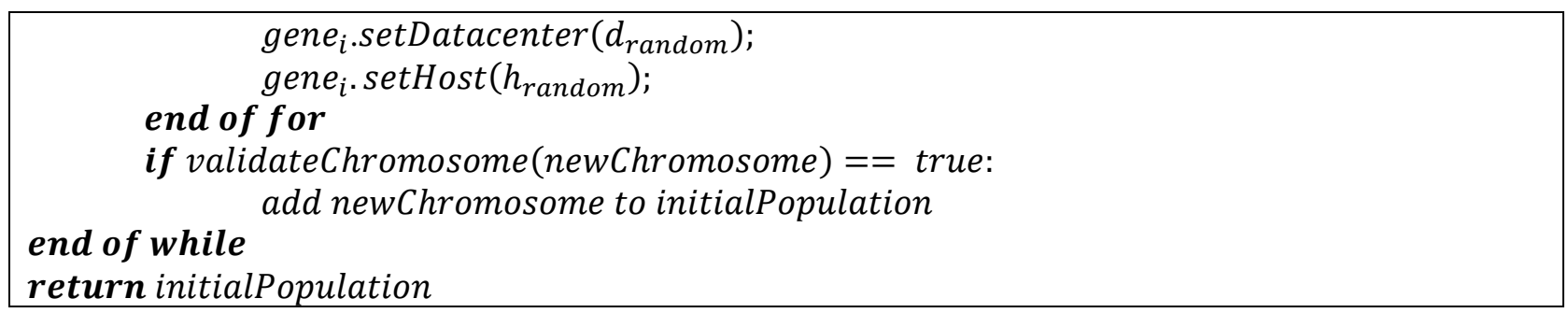

\section{4-1-2- Selection Operator}

The selection process causes the chromosomes to have a better fit. For the selection operator, different methods are used, such as the roulette wheel or the binary tournament. In the roulette wheel method, the whole population is divided into some sections, each of which represents an individual. The probability of choosing an individual for the next generation is determined by the ratio of the individual's fitness value to the total fitness values of the entire population. In contrast, in the binary tournament method, the individual with the highest fitness value is selected for the next generation. Here, there are no arithmetic calculations based on the value of proportionality. The selection of an individual for the next generation is based solely on several comparisons between individuals based on the value of fitness. Usually, the speed of convergence of the tournament strategy is much faster than the roulette wheel. Algorithm 6 provides the pseudo-code for selecting individuals based on tournament strategy. First, a subset of the attributes of a population is selected. Then, members of the population compete with each other, and ultimately only one attribute from each subgroup is selected. The tournament method provides fair conditions for the selection of all solutions and thus preserves diversity.

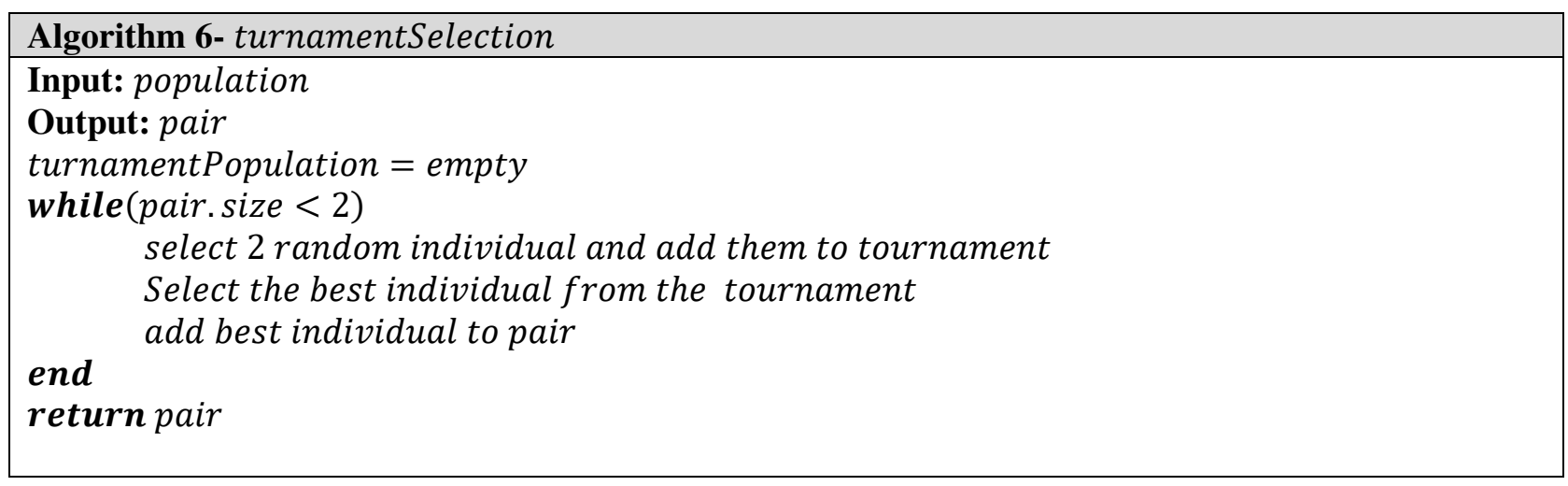

Before definitively producing each chromosome, we check the limitations in Eqs. (19)-(20). Pseudo-codes of chromosome validation are provided in Algorithm 7 and Algorithm 8.

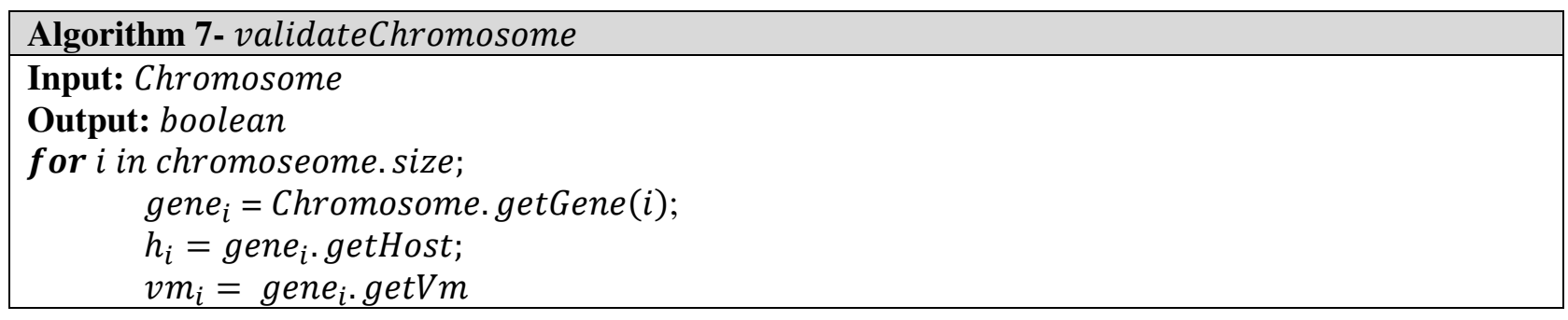




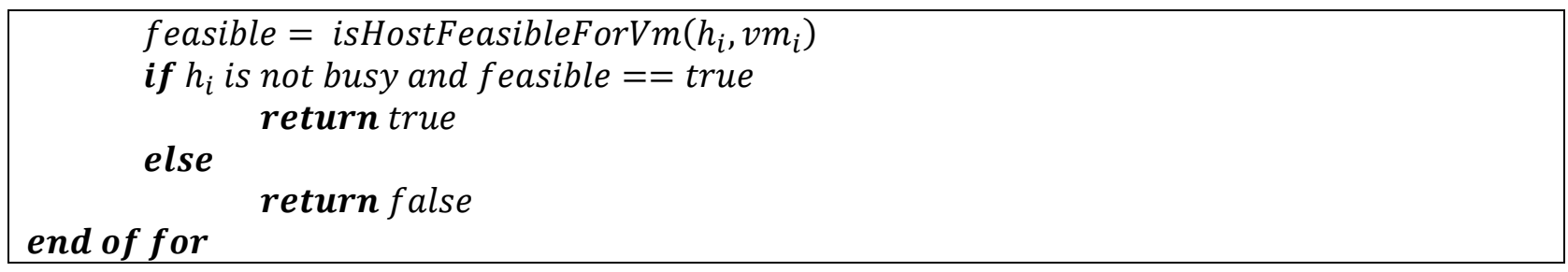

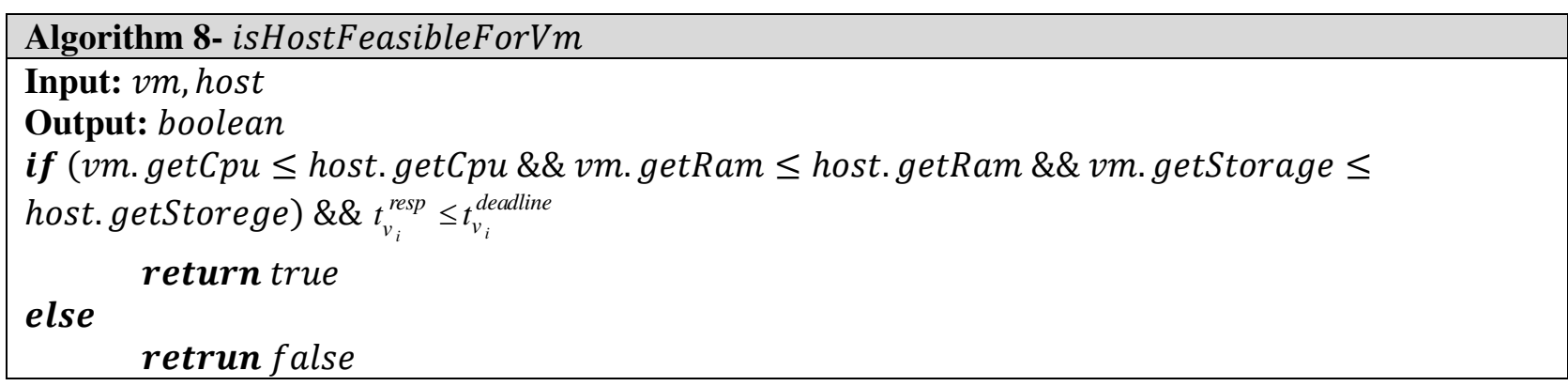

\section{4-1-3- Cross-over Operator}

During the cross-over process, parts of the parent chromosomes are randomly swapped. This allows offsprings (children) to inherit a combination of their parents' attributes. The chromosomes that are selected for the crossover operation and the resultant chromosomes are called "parent" and "child", respectively. According to Fig. 3, based on experimental observations, we considered the appropriate cross-over rate to be 0.7 . In this study, we use a two-point method for the cross-over operation. This operator randomly selects two points from the parent chromosomes and swaps the genes of those points. The pseudo-code of the cross-over operation is shown in Algorithm 9.

\begin{tabular}{|l|}
\hline Algorithm 9- crossover \\
\hline Input: pair \\
Output: off spring chromosomes \\
generate two random positions \\
swap data beyond the two points between chromosom in pair \\
return pair
\end{tabular}




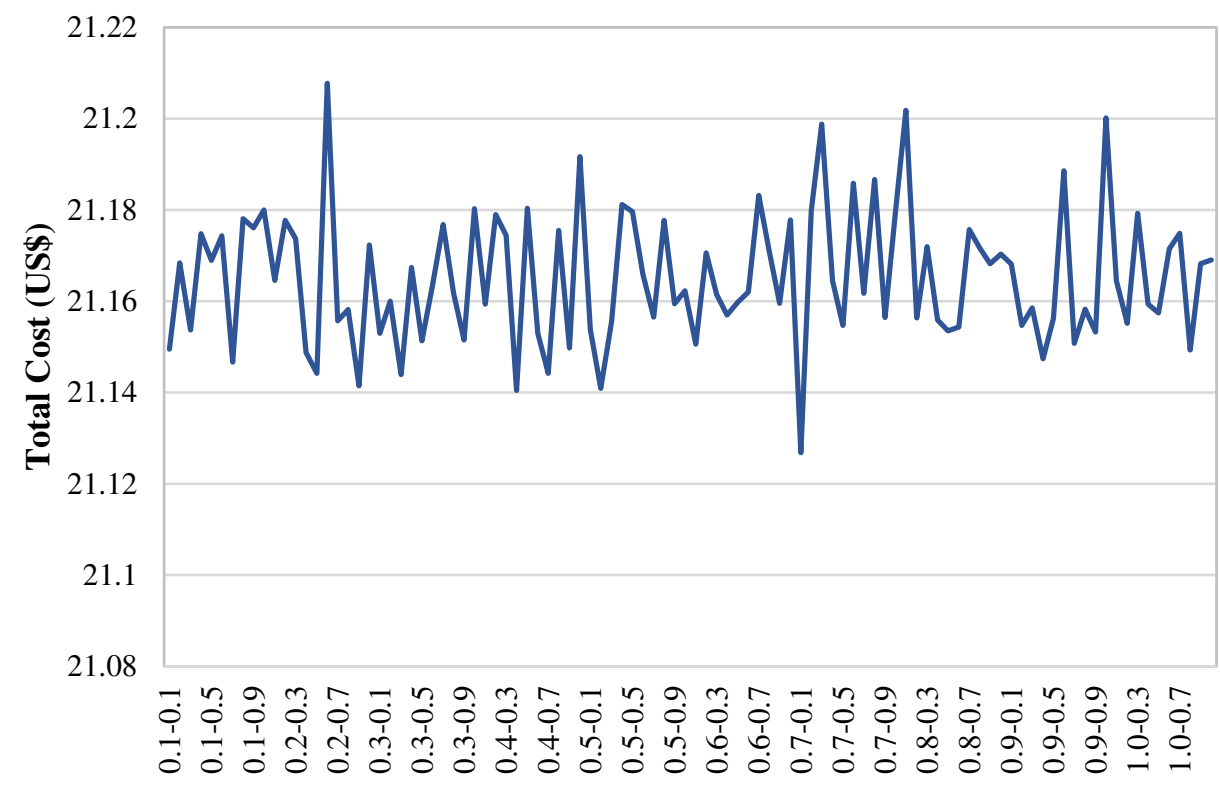

Cross-over Rate vs. Mutation Rate

Fig. 3- Impact of cross-over rate and mutation rate on overall costs

\section{4-1-4- Mutation Operator}

A mutation operator is used to increase genetic diversity in a population and, consequently, to increase the chances of achieving an optimal solution in a shorter time. This operator also causes the algorithm to avoid getting stuck in the optimal local solutions. This operator modifies one or more genes in a chromosome with a small probability called the mutation probability. Like the crossover operator, here we assume a mutation probability of 0.1 based on the experimental observations shown in Fig. 3. Selecting this value resulted in a more stable response than other values. As shown in Fig. 3, selecting this value also causes fewer fluctuations in the cost function. The pseudo-code of the mutation routine is shown in Algorithms 10 and 11. As shown in the pseudo-code of Algorithm 12., the crossover operation is performed with a probability of $P_{\text {crossover }}$ and the mutation operation is performed with a probability of $P_{\text {mutation }}$, and ultimately the population size reaches populationSize.

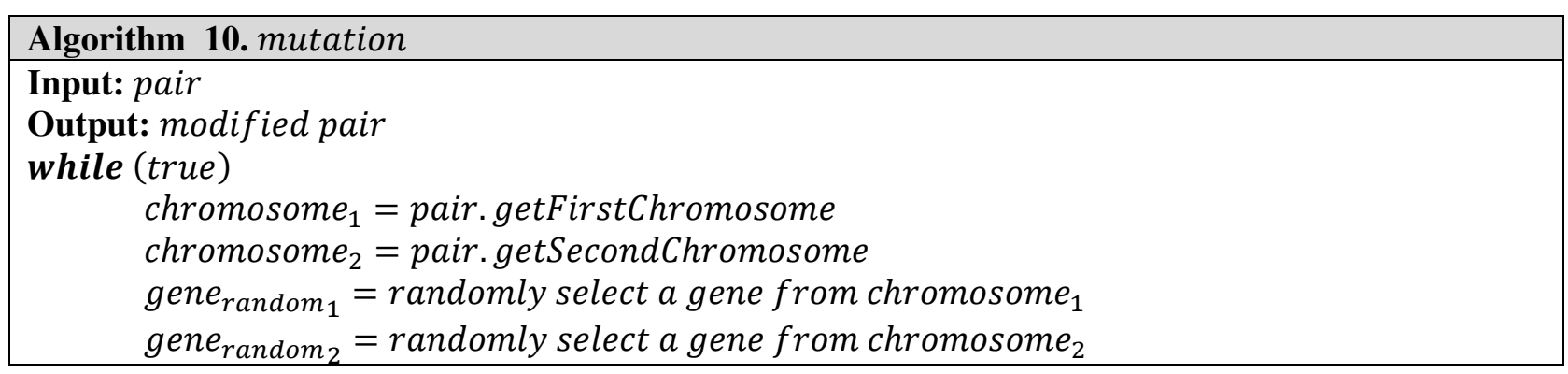



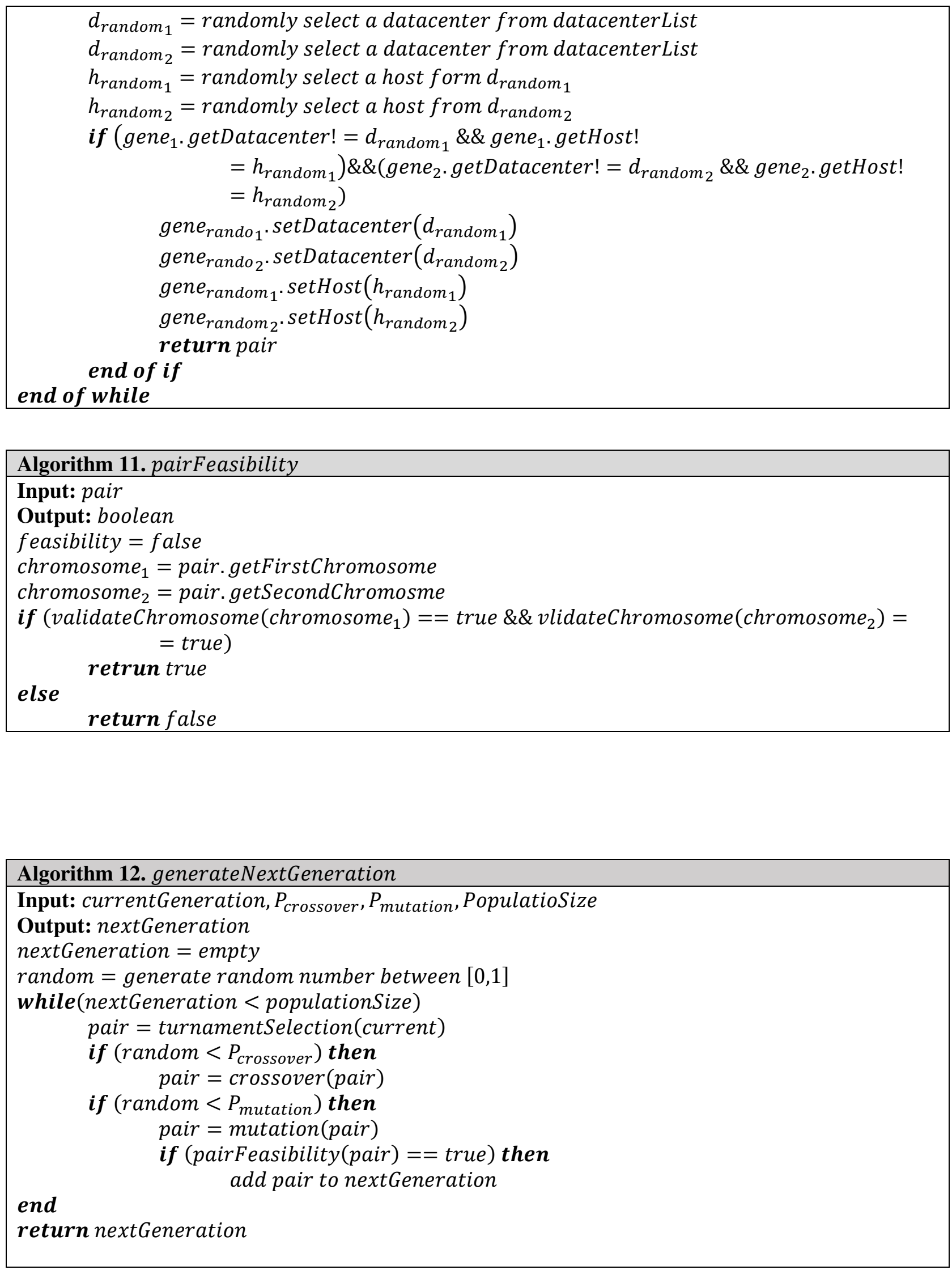


\section{4-1-5- The Fitness Function}

In the GA, the idea is to remove the $\mathrm{N}$-worst possible solutions (chromosomes) and leave the most appropriate ones. To measure the quality of solutions, we use a function called the fitness function. The larger the value of this function, the higher the quality of the solution. The fitness function was previously shown in Eq. (24) in which our goal is to minimize the cost. The pseudo-code for calculating chromosome fitness is shown in Algorithms 13 and 14.
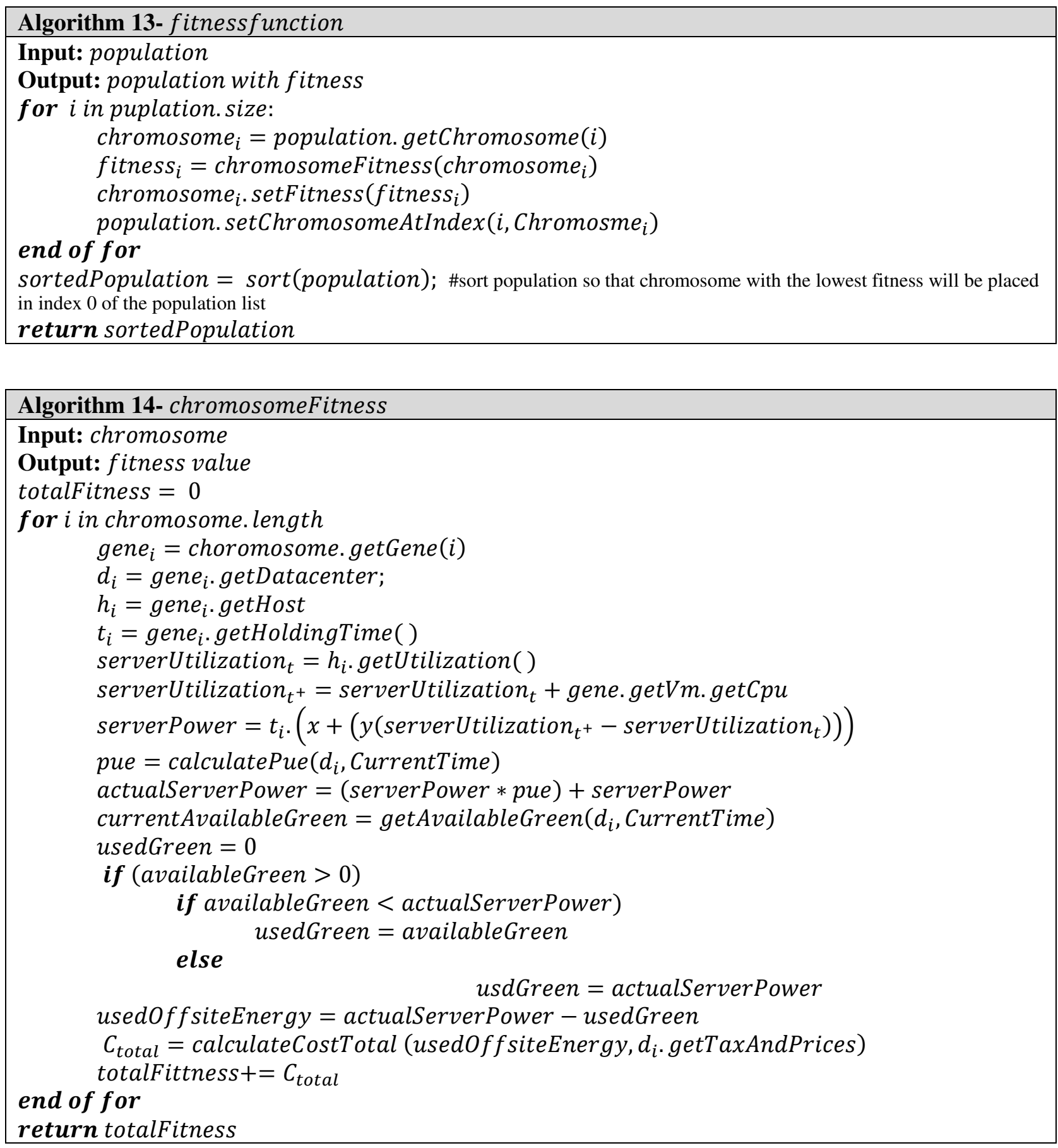
As shown in Algorithm 14, the server utilization at an upcoming round $t^{+}$is the sum of server utilization and the amount of required CPU. We have already obtained server power consumption in Eq. (2) as a function of CPU utilization. Also, according to Eq. (5), the total consumed energy is equal to the total energy consumed by the server and the energy consumption overhead. The PUE metric mentioned in Eq. (1) is used to calculate the overhead. In the final rows of Algorithm 14, the amount of carbon dioxide is checked. Then, according to the type and the amount of consumed energy and tax costs, the amount of costs imposed on the system is calculated. If the amount of solar energy is less than the required energy of the VM, the server will use all available solar energy and consumes the rest of its energy needs from an offsite energy source. As stated in Eq. (3)-(4), the energy cost of each data center includes two parts: the cost of consumed energy and the cost that is incurred due to carbon production. The total cost of the data center is obtained by adding the total amount of consumed energy, operational costs, the amount of carbon dioxide, and the cost of the tax. For each chromosome, this value is added to the fitness value. The above calculations are performed for all genes.

\section{4-2- Memetic Algorithm}

\section{4-2-1- Main Algorithm}

The GA requires a lot of time to process different generations to achieve the desired result [14]. To overcome this shortcoming, we present a GA-inspired algorithm called the Mimetic Algorithm (MA). We call this method, "Cost and Renewable Energy-Aware Dynamic PUE with Memetic Algorithm" or in abbreviation CRA-DP-MA. The structure of each possible solution is similar to what was shown previously in Fig. 1. Unlike the GA, each chromosome in the MA, once created, tries to improve its fitness by using a local search method, such as Hill Climbing [37]. The MA flowchart is similar to what we showed earlier in Fig. 2, except that in the final stage, each chromosome (a possible solution) finds its neighbor solutions through minor modifications in its current state. It then replaces the best neighbor chromosome with the worst solution in the population [14]. Our goal is to minimize the cost function in Eq. (24). Since the selection, crossover, and mutation routines in the MA are the same as the GA, we do not show them again. Also, we consider the climbing rate to be 0.8 based on the experiments shown in Fig. 4. The pseudo-code of the CRA-DP-MA is shown in Algorithm 15 and its flowchart is depicted in Fig 5. Note that the probability of crossover, mutation, and local search is denoted by $P_{\text {crossover }}$, $P_{\text {mutation }}$, and $P_{\text {localsearch }}$, respectively. 


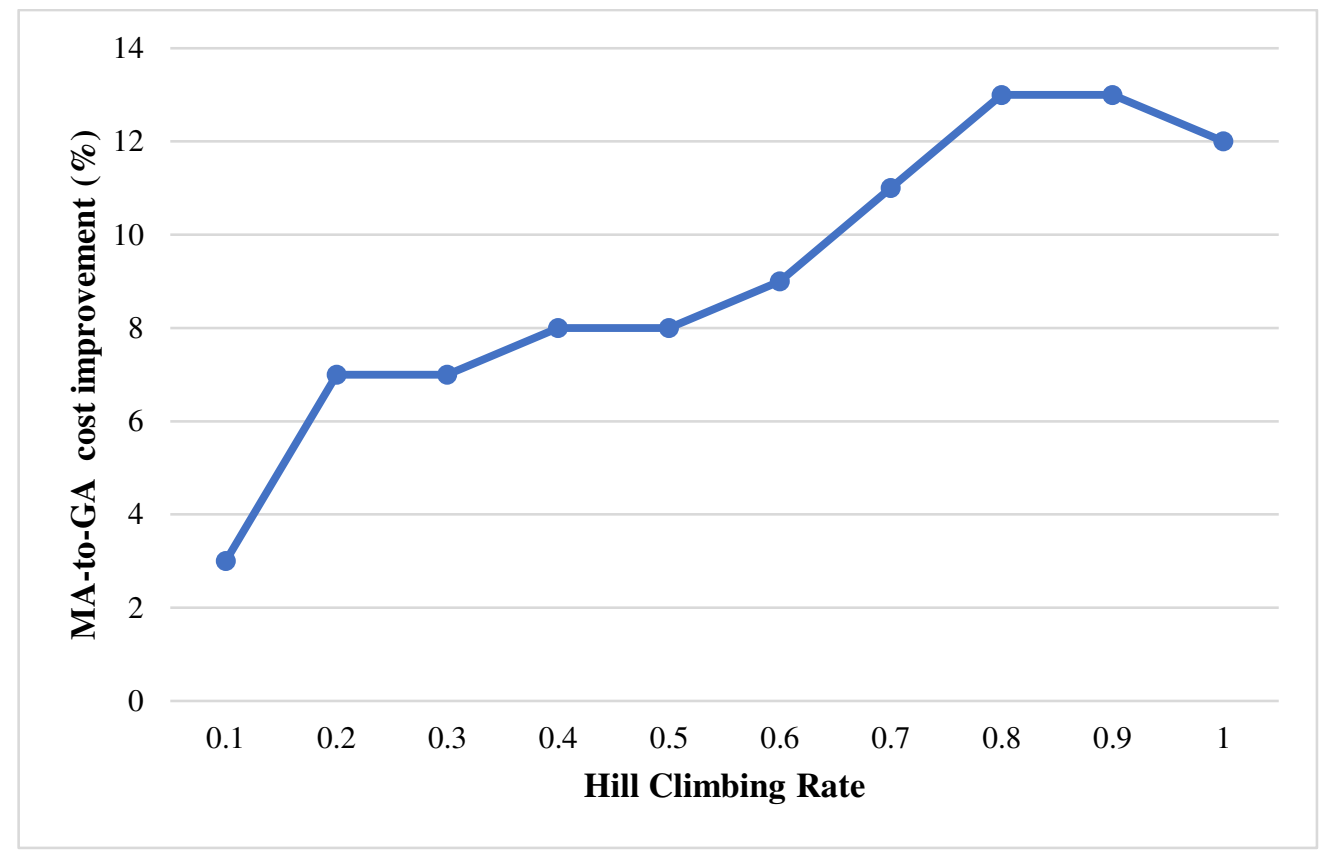

Fig. 4 - Amount of objective function improvement in the CRA-DP-MA against the CRA-DP-GA for different values of climbing rates 


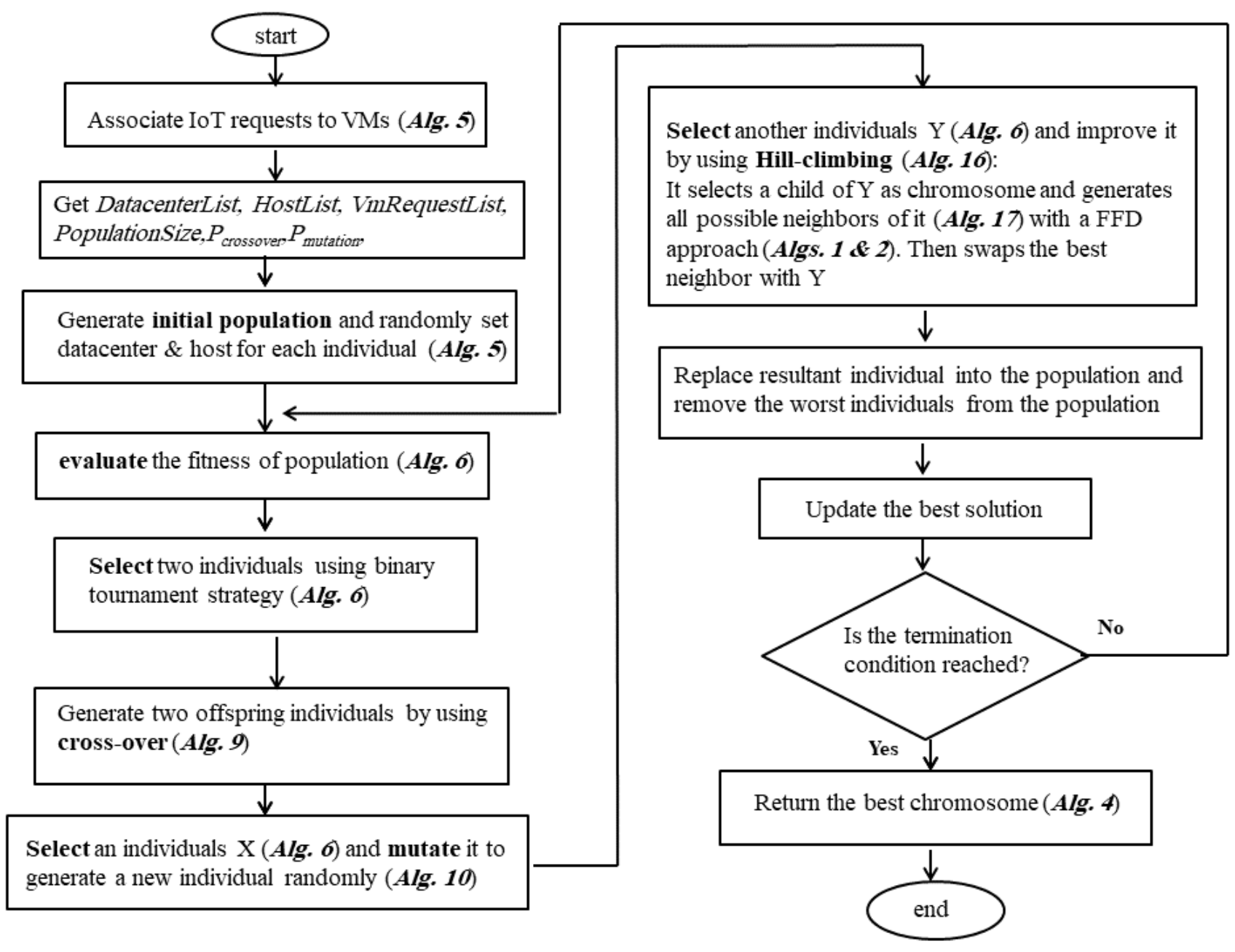

Fig. 5- Flowchart of the CRA-DP-MA

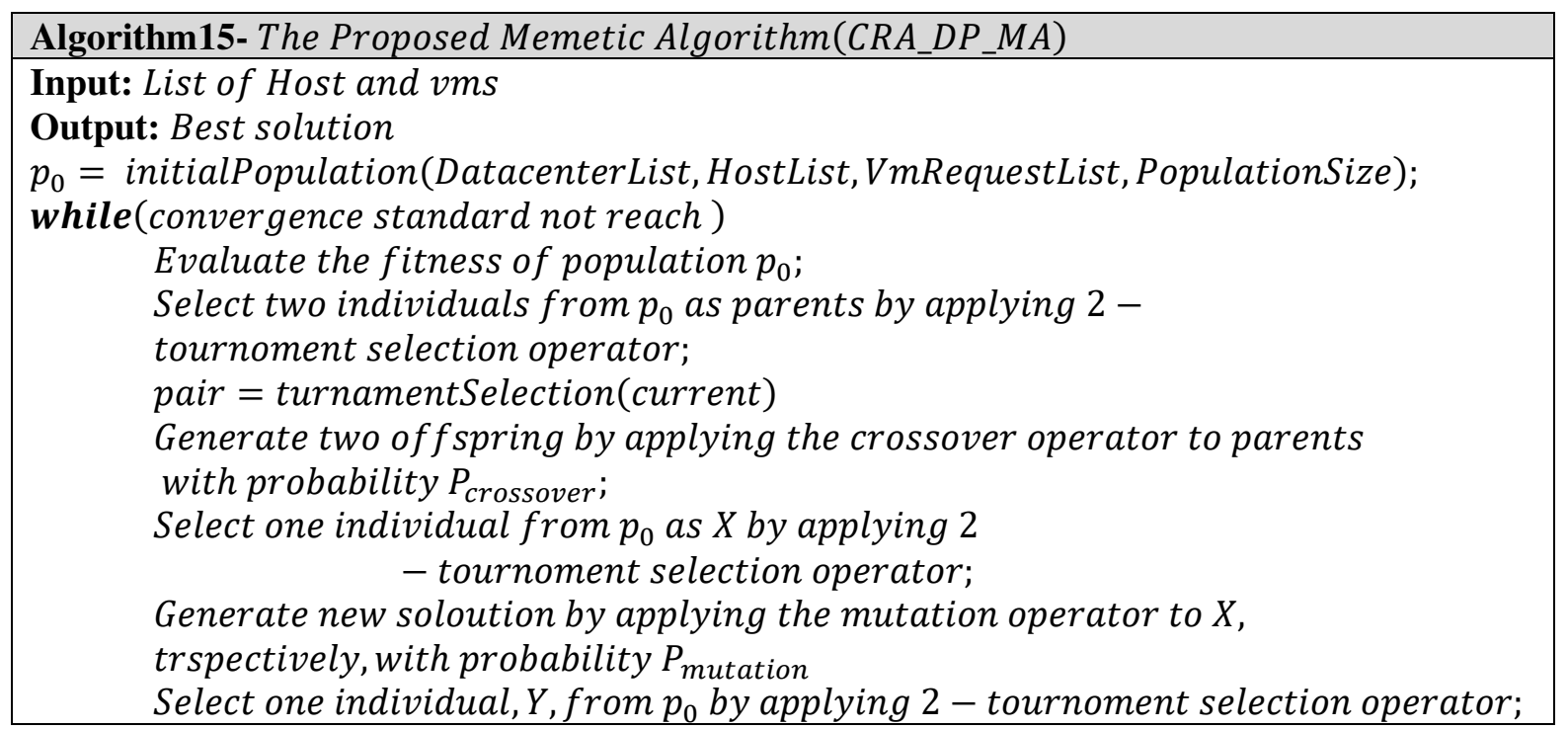




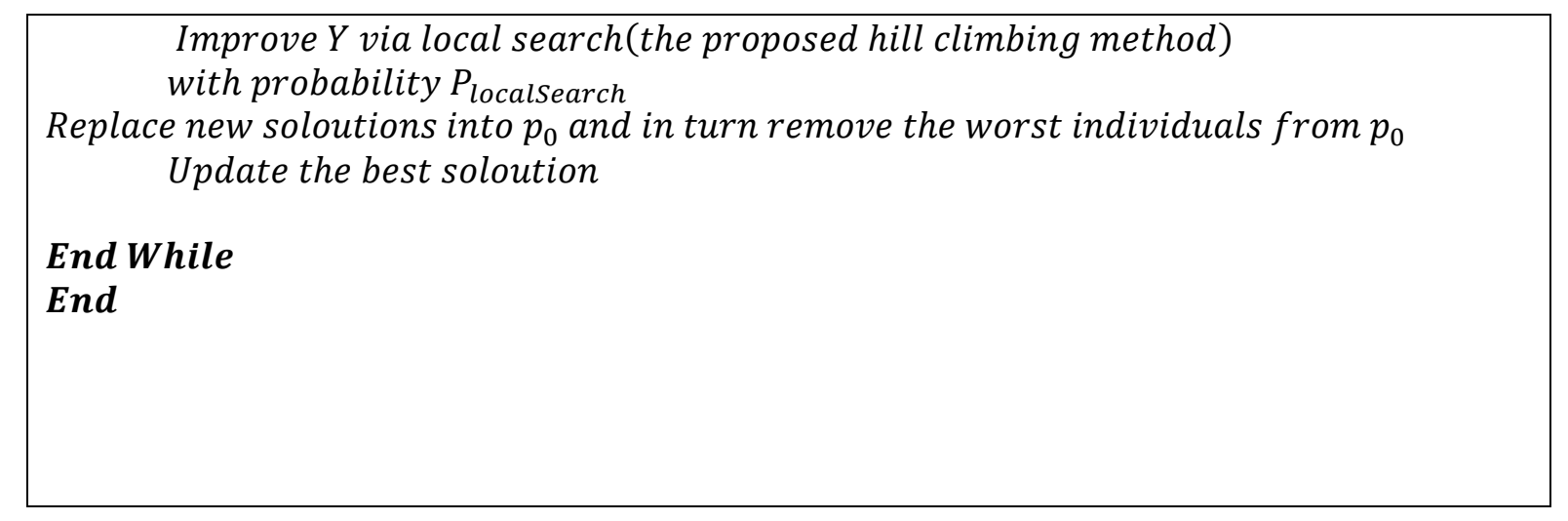

\section{4-2-2- Local Search}

As explained in section 4-2-1, the major difference between the MA and the GA is local search, for example, a hill-climbing method. As shown in Fig. 6, hill-climbing is an iterative technique that starts with a candidate solution and then tries to achieve a better solution by moving on neighbors [37]. If this movement leads to a better solution, another move will be made on this new solution. This process continues until further improvement in the solution space is not possible. It can be said that the hill-climbing method tries to minimize the objective function of Eq. (24), namely $c^{\text {total }}$. In each iteration, it selects an element of the state space, i.e. vector $\mathbf{Z}_{N_{V} \times N_{H}}$, and examines whether this change improves the value of the objective function. This process continues until no change leads to an improvement in the value of $c^{\text {total }}$. At this point, the vector $\mathbf{Z}_{N_{V} \times N_{H}}$ will be the "local minimum" and the output of the algorithm. The most significant reasons for terminating the algorithm are the existence of a local minimum, flat, and ridges. Since each candidate solution can have multiple neighbors, the heuristic by which neighbors are selected is crucial.

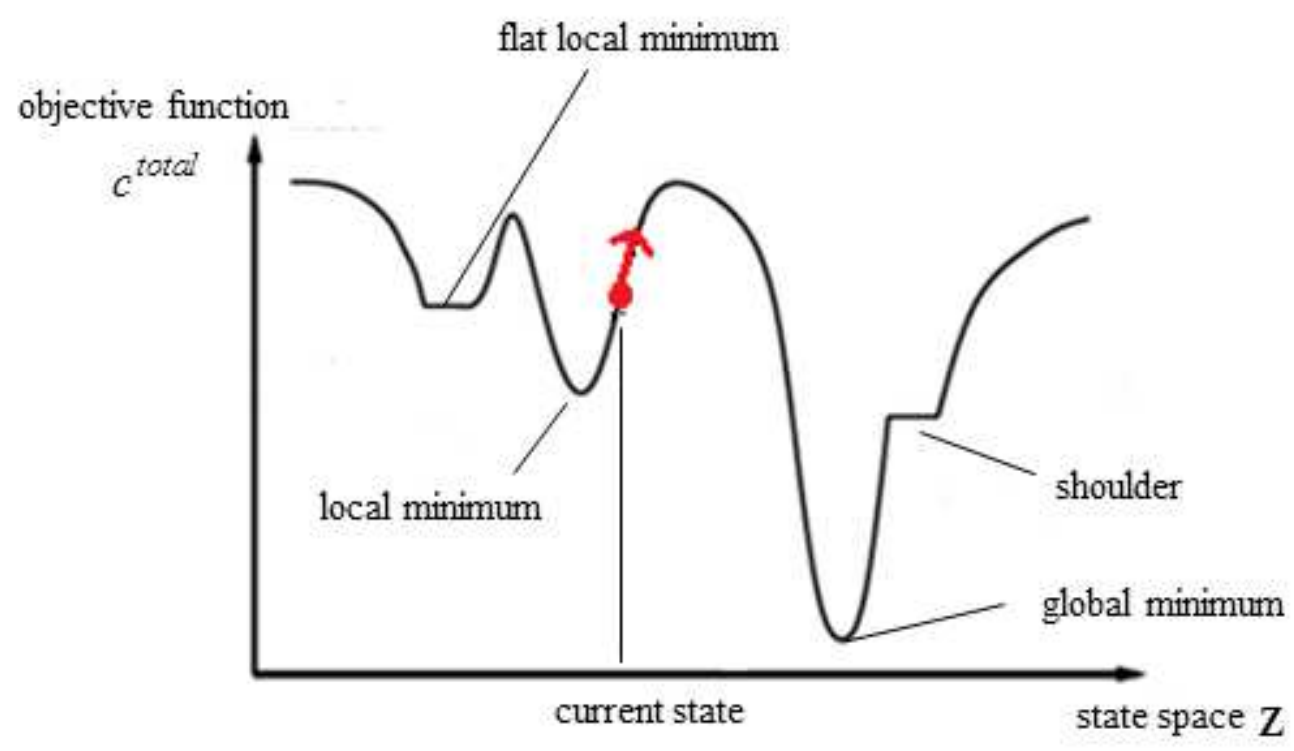

Fig. 6- state-space in the local hill-climbing search algorithm 
We propose a method called Host-based Hill-climbing, the pseudo-code of which is shown in Algorithm 16. The only factor that limits the performance of the hill-climbing algorithm is the number of neighbors. If the number of neighbors is large, the cost of this method may be very high [14]. To solve this problem, we have defined a method called Host-based Neighborhood ( $\mathrm{HbN})$ in Algorithm 17. In Algorithm 16, for each gene, we call Algorithm 17. Finally, Algorithm 16 generates all possible neighbors with the FFD algorithm, without placement. Simply speaking, in the process of finding neighbors, if the host for a gene is already selected, there is no chance of re-election. Eventually, the best neighboring chromosome is replaced by the current chromosome.
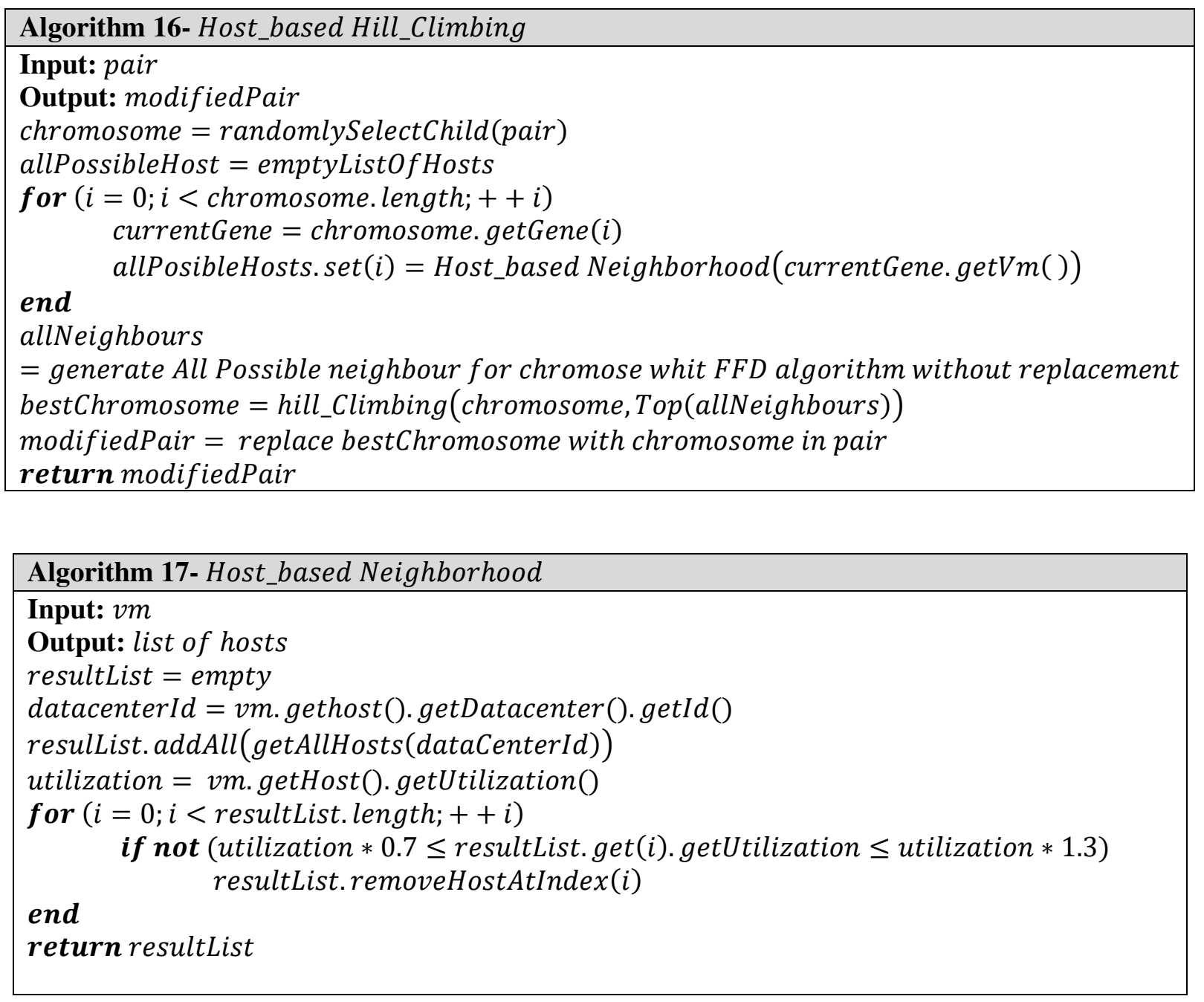

We now proceed to describe the decisions made at each step of Algorithm 17.

Step 1: As shown in Fig. 7, the algorithm must first find all available hosts in the same data center for each gene. For example, if gene \#1 on chromosome \#X is located inside datacenter \#4, we need to inspect all the hosts inside datacenter \#4. The reason why we inspect only the hosts in the current data center is that the locality of the solution must be met. If we use the hosts inside other data centers, it leads to diversity in temperature conditions, and consequently the availability of renewable energy. 


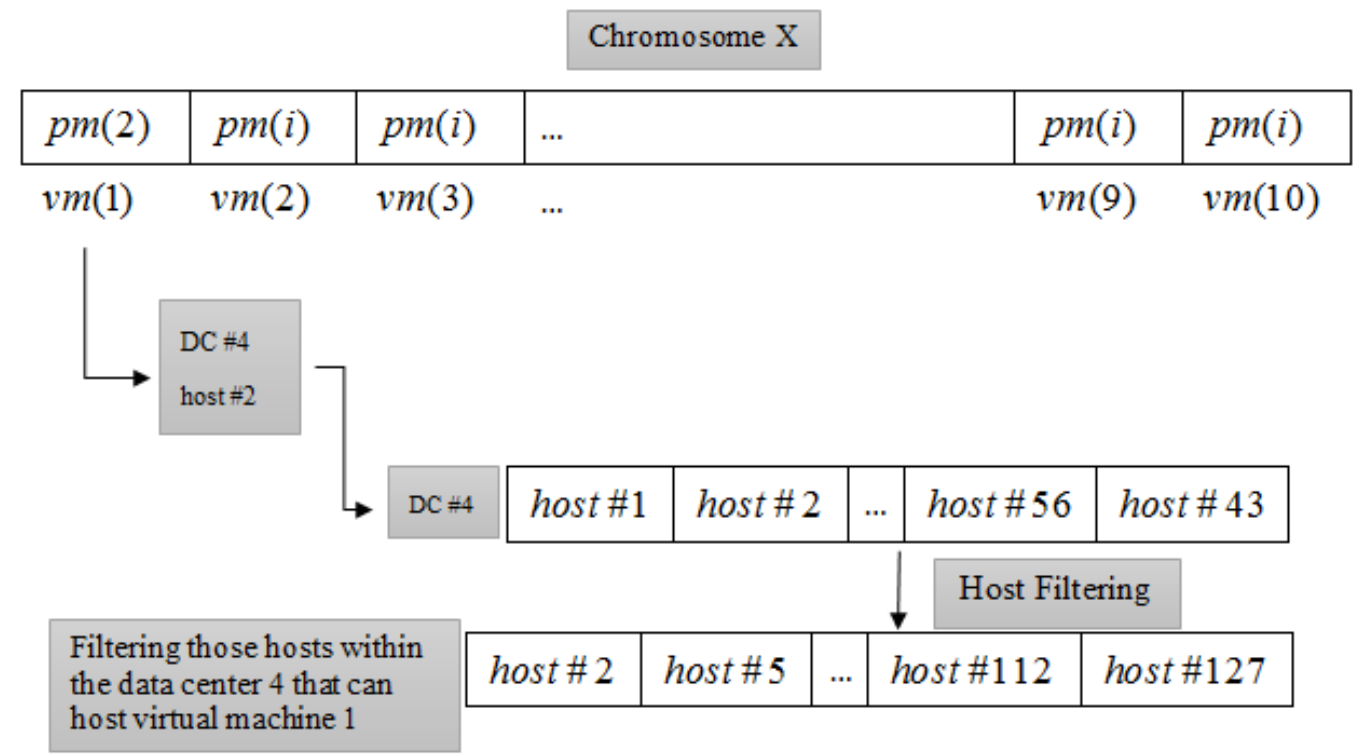

Fig. 7- Finding potential hosts in the Host_based Neighborhood $(\mathrm{HbN})$ algorithm

Step 2: Once the permissible hosts for each gene have been found, those with the same utilization should be selected as the current host. Based on the experimental results shown in Fig. 8, we set the threshold for utilization similarity to be $30 \%$. Based on this, a host that has at least $30 \%$ similarity with the current host is selected. Otherwise, the host will be removed from the neighbors' list. This decision balances the load and also prevents allocations from being biased towards empty or overloaded hosts.

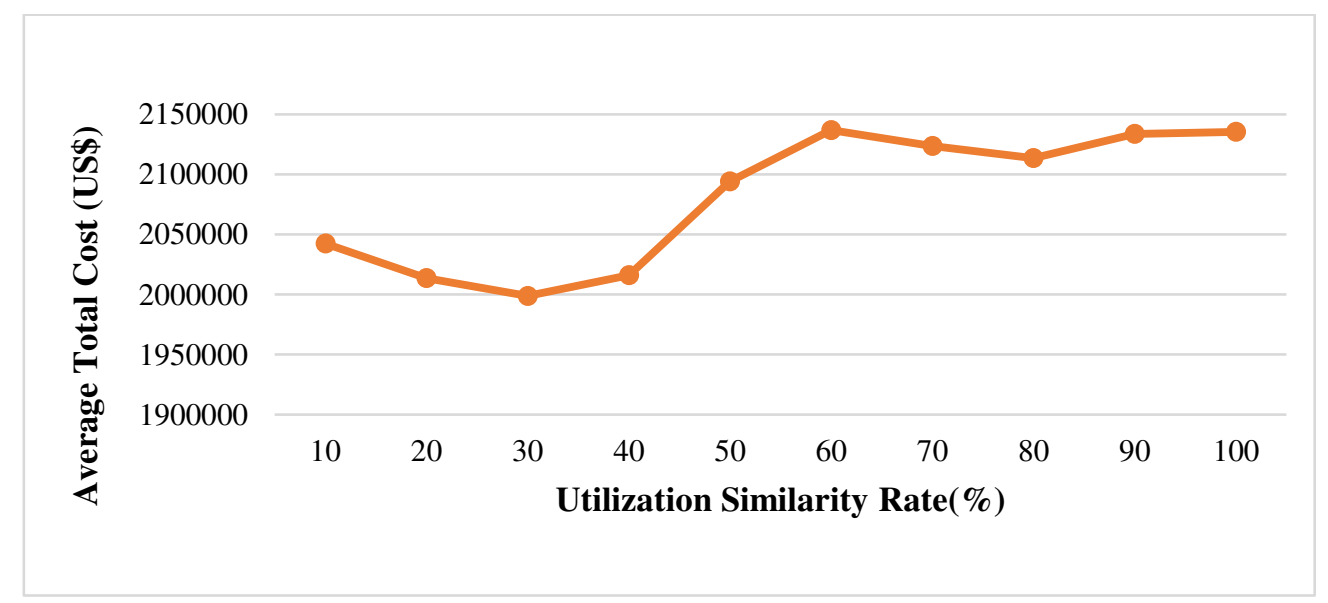

Fig. 8- Average total cost vs. utilization similarity rate for 30 runs

Step 3: In this step, for each gene, several candidate hosts are obtained, each of which can identify the candidate neighbors. We then randomly select a maximum of 10 different neighbors using the FFD algorithm without replacement. Experimental results show that the optimal value for the number of neighbors in a local search routine is 10 . If we consider the number of neighbors more than 10 , the efficiency of the algorithm decreases. 
Conversely, if we consider the number of neighbors more than 10 , there is no significant improvement in the solution.

Step 4: In this step, the local search algorithm is applied to 10 neighbors obtained from the previous step and the best solution is selected.

\section{4-3- CRA-DPSCH-DDMPEA method}

We compare our proposed method with one of the state-of-the-art methods proposed by Chauhan et al. [38]. The method is called the diversity-driven multi-parent evolutionary algorithm (DDMPEA) with adaptive nonuniform mutation (ANUM). In the diagrams and algorithms, the implementation of the joint cost and scheduling optimization problem (Eq. (36)) using this method is abbreviated as CRA-DPSCH-DDMPEA. For a fair comparison with state-of-the-art methods, we will use the DDMPEA later in Section 5 for the sub-problem of Eq. (24). In the CRA-DPSCH-DDMPEA, non-uniform mutations are used to maintain diversity in solutions [39]. Here, fitness variance is used to detect premature convergence of the population to local optima. Also, more than two parents are used for cross-over operations. After the selection of several parents and cross-over, a non-uniform adaptive mutation occurs, which in turn results in a reduction in the variance of the candidate solutions. Hence, it can be said that the algorithm is driven according to population diversity. This ensures that it does not get stuck in the local optima. The steps of CRA-DPSCH-DDMPEA are described in detail as follows:

\section{- Population Initialization}

Initially, $N_{P}$ members are randomly generated according to the lower and upper bounds of the search space using a uniform distribution as follows:

$$
x_{i j}=x_{j}^{\min }+r_{i j}\left(x_{j}^{\max }-x_{j}^{\min }\right), \quad i=1,2, \ldots, N_{P} ; j=1,2, \ldots, D
$$

, where $r_{i j}$ is a random number with uniform distribution for member $i$ and dimension $j$. Here, $N_{P}$ is the

population size and $D$ is the search space dimension. Also, $x_{j}^{\min }$ and $x_{j}^{\max }$ are the minimum and maximum for the variable.

\section{- Multi-parent Cross-over}

Unlike GA, here, three parents are used for cross-over. For this purpose, a set of parents is first selected from the best $p \%$ of the total population. After selecting three random members $x_{r_{1}}, x_{r_{2}}$, and $x_{r 3}$ from this set, an offspring $O_{i j}^{t}$ is created with the following formula:

$$
O_{i j}^{t}=x_{r 1, j}^{t}+\alpha_{i j}\left(x_{r 2, j}^{t}-x_{r 3, j}^{t}\right), \quad i=1,2, \ldots, \frac{1}{3} \cdot N_{P} \cdot \frac{p}{100} ; j=1,2, \ldots, D
$$

, where $\alpha_{i j}$ is a random weight that follows a normal distribution with a mean of 0.7 and a variance of 0.1 . Also, $t$ represents the number of generations produced so far. In this technique, Differential Evolution (DE) capabilities are used [36]. 
The control operation is now performed on the generated offspring to ensure that it is within the allowable range:

$$
O_{i j}^{t}= \begin{cases}\frac{O_{i j}^{t}+x_{j}^{\min }}{2}, & \text { if } O_{i j}^{t}<x_{j}^{\min } \\ \frac{O_{i j}^{t}+x_{j}^{\max }}{2}, & \text { if } O_{i j}^{t} \geq x_{j}^{\max }\end{cases}
$$

Now, the fitness of the created offspring is assessed by placing the answer in Eq. (43).

\section{- Adaptive Mutation Strategy}

As is common in evolutionary computing, mutation are used to maintain diversity from one generation to the next [40]. If the algorithm gets stuck in a local optimum, then the variance of the population fitness will become zero. To cope with his situation and also to create faster convergence, we use an adaptive non-uniform mutation operator as follows:

$$
O m_{i j}^{t}=O_{i j}^{t}(1+0.5 \eta), \quad i=1,2, \ldots, \frac{1}{3} . N_{P} \cdot \frac{p}{100} ; j=1,2, \ldots, D
$$

, where $\eta$ denotes the weight coefficient obtained from a normal distribution with a mean 0 and a variance of 1 . The mutation occurs when $r_{i}(0,1)<p_{m}$, where $r_{i}(0,1) \in U(0,1)$ is a uniform random number in the interval $(0,1)$ for the $i$-th offspring. Also, $p_{m}$ is the probability of mutation that results from the following equation:

$$
p_{m}=\left\{\begin{array}{cc}
\frac{e^{-h}}{5}, & \text { if } \sigma^{2}<\sigma_{1} \\
0, & \text { if } \quad \sigma^{2} \geq \sigma_{1}
\end{array}\right.
$$

, where $\sigma_{l}$ is the variance threshold, which is calculated from the range of variables as follows:

$$
\sigma_{1}=\frac{1}{100}\left(x_{j}^{\max }-x_{j}^{\min }\right), j=1,2, \ldots, D .
$$

Also, $\sigma^{2}$ denotes the variance of population fitness. Here, the degree of diversity in the iteration $t$ is obtained as follows:

$$
h=\frac{\max _{1 \leq i \leq N_{P}}\left\{x_{i, j}^{t}-x_{\text {best } 2}^{t}\right\}}{\max _{t}\left\{\max _{1 \leq i \leq N_{P}}\left\{x_{i, j}^{t}-x_{\text {best } 2}^{t}\right\}\right\}}
$$

, where $x_{\text {best } 2}^{t}$ is the position of the best fitness value in the iteration $t$. When the value of $h$ is high, individuals become more scattered in the search space, so a small amount of mutation is needed. Conversely, when the value of $h$ is small, individuals become more crowded in the search space, and therefore a large amount of mutation is needed. Roughly speaking, when the CRA-DPSCH-DDMPEA gets stuck in local minima, it tries to escape this bad situation by leveraging the fitness variance. This parameter is calculated as follows: 


$$
\sigma^{2}=\sum_{i=1}^{N_{P}}\left(\frac{f_{i}-\bar{f}}{f}\right)^{2}
$$

, where $f_{i}$ is the fitness of $i$-th individual and $\bar{f}$ is the mean of the fitness values. Also, $f$ is the returning factor used to control the individuals' fitness variance and is calculated as follows:

$$
f=\left\{\begin{array}{cl}
\max \left|f_{i}-\bar{f}\right|, & \text { if }\left|f_{i}-\bar{f}\right|<1 \\
1, & \text { otherwise }
\end{array}\right.
$$

The fitness variance $\sigma^{2}$ indicates population density. The smaller it is, the closer individuals are assembled. Large amounts of fitness variance indicate that individuals are randomly dispersed. Once the mutation is done, the new individual is added to the population. The population is then ranked according to the fitness value of the individuals. Finally, $N_{P}$ best individuals are selected and create the next-generation population. Before proceeding, it should be noted that various methods have been used by researchers to achieve diversity. Interested readers can refer to [41] for further study.

As previously stated in Eqs. (36)-(40), the optimization problem in this study has both inequality constraints (denoted by $g_{i}(\mathrm{X})$ ) and equality constraints (denoted by $h_{i}(\mathrm{X})$ ). Let us denote the number of inequality and total constraints by $G$ and $M$, respectively. Thus, they can be shown as follows:

$$
\begin{gathered}
g_{i}(\mathrm{X}) \leq 0, \quad i=1, \ldots, G . \\
h_{j}(\mathrm{X})=0, \quad j=G+1, \ldots, M .
\end{gathered}
$$

For the sake of convenience, we can combine the above two constraints and rewrite them as follows:

$$
G_{i}(\mathrm{X})=\left\{\begin{array}{cl}
\max \left\{0, g_{i}(\mathrm{X})\right\}, & i=1, \ldots, G \\
\max \left\{0,\left|h_{i}(\mathrm{X})\right|-\delta\right\}, & i=G+1, \ldots, M
\end{array}\right.
$$

To obtain the total constraint violation $V(\mathrm{X})$ for an infeasible solution, we calculate the weighted average of all constraints as follows:

$$
V(\mathrm{X})=\sum_{i=1}^{M} G_{i}(\mathrm{X})
$$

Decision theory is used to manage constraints in the optimization problem [42]. Here, there are many methods, one of the most common of which is fuzzy decision-making. In this method, the input variables are fuzzified into a membership function $\mu$. Here, each input variable $X$ is assigned a membership value in the range $[0,1]$. A value of $\mu=0$ for an input variable indicates that it is deterministic, not fuzzy. Simply speaking, the larger the membership value $\mu$ for an input variable $X$, the fuzzier it is. The fuzzy membership function $\mu$ is plotted in Fig. 9 and is expressed as follows: 


$$
\mu\left(F_{i}\right)=\left\{\begin{array}{cc}
1, & \text { if } F_{i} \leq F_{i}^{\min } \\
\frac{F_{i}^{\max }-F_{i}}{F_{i}^{\max }-F_{i}^{\min },} & \text { if } F_{i}^{\min }<F_{i}<F_{i}^{\max }(i=1,2) \\
0, & \text { if } F_{i} \geq F_{i}^{\max }
\end{array}\right.
$$

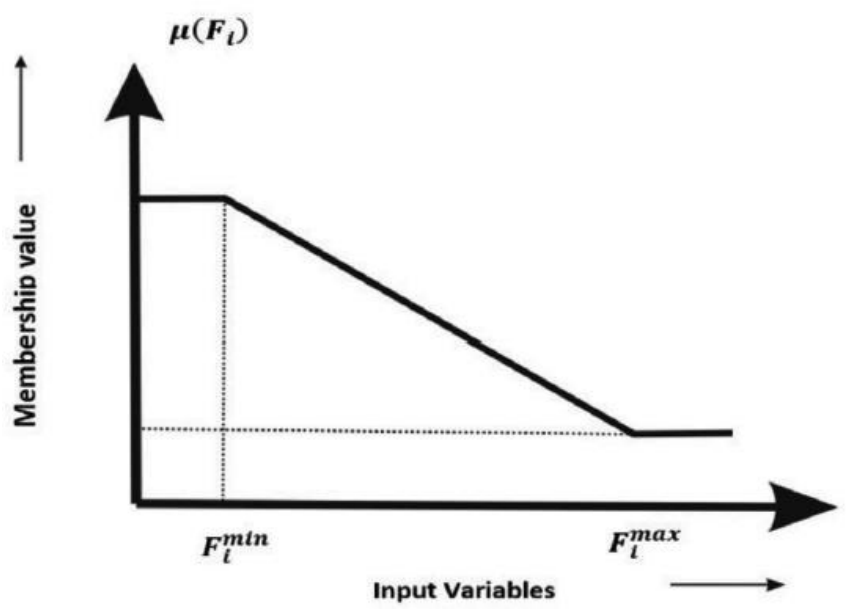

Fig. 9- Fuzzy membership function in this research

It is important to note that here we have two objective functions, $F_{1}$ and $F_{2}$. The objective function $F_{1}$ is the same as our principal objective as stated in Eq. (36). The objective function $F_{2}$, imposed by fuzzy decisionmaking, indicates the degree of constraint violation. The value of the membership function indicates the level of satisfaction of the objective function $F_{i}$. Let us denote the number of all non-dominated solutions by $K$. We now proceed to rank the achievement of a non-dominated solution $k$ concerning all solutions. To do this, we must first divide the achievement of this solution by the sum of the achievements of all non-dominated solutions. By performing such normalization, the function $\mu_{D}^{k}$ can be calculated as a membership function for nondominated solutions as follows:

$$
\mu_{D}^{k}=\frac{\sum_{i=1}^{M} \mu\left(F_{i}^{k}\right)}{\sum_{k=1}^{K} \sum_{i=1}^{M} \mu\left(F_{i}^{k}\right)}
$$

The best solution $O_{\text {best }}$ is an offspring that manages to get the maximum membership score $\mu_{D}^{k}$. It is obtained as follows:

$$
O_{\text {best }}=\max \left\{\mu_{D}^{k}: k=1,2\right\}
$$

The pseudo-code of the CRA-DPSCH-DDMPEA is shown in Algorithm 18. 
Input: number of individuals $N_{P}$, the search space dimension $D$, principal objective function $F_{1}$, inequality constraints $g_{i}(\mathrm{X})$, equality constraints $h_{i}(\mathrm{X})$, a ratio for accepted solutions $p$

Output: The best solution $O_{\text {best }}$

Create the initial population containing individuals like $x_{i j}$ using Eq. (41).

Evaluate the objective function $F_{1}\left(x_{i j}\right)$

Evaluate the constraint violation $F_{2}=V(\mathrm{X})$ using Eq. (53).

Evaluate the fuzzy membership function $\mu\left(F_{i}\right)$ using Eq. (54).

Evaluate normalized membership function $\mu_{D}^{k}$ using Eq. (55).

Identify the best solution $O_{\text {best }}$ using Eq. (56).

for $t=1$ to $T$

Evaluate the objective function $F_{1}\left(x_{i j}\right)$

Evaluate the constraint violation $F_{2}=V(\mathrm{X})$ using Eq. (53).

Evaluate the fuzzy membership function $\mu\left(F_{i}\right)$ using Eq. (54).

Evaluate normalized membership function $\mu_{D}^{k}$ using Eq. (55).

Sort the whole population based on $\mu_{D}^{k}$ values and select the best $p \%$.

for $i=1$ to $(1 / 3) \cdot N_{P} \cdot(p / 100)$

Select three random members $x_{r 1}, x_{r 2}$, and $x_{r 3}$

Select the random weight $\alpha_{i j}$ using a normal distribution $N(0.7, \sqrt{0.1})$.

Create an offspring $O_{i j}^{t}$ using the cross-over operation of Eq. (42).

Perform the control operation on the new offspring $O_{i j}^{t}$ using Eq. (43).

\section{end for}

Evaluate the objective function $F_{1}\left(O_{i j}^{t}\right)$

Evaluate the constraint violation $F_{2}\left(O_{i j}^{t}\right)=V\left(O_{i j}^{t}\right)$ using Eq. (53).

Calculate the fitness variance of the population using Eq. (48).

Calculate the degree of diversity, $h$, using Eq. (47).

Calculate the probability of mutation, $p_{m}$, using Eq. (45).

if $r_{i}(0,1)<p_{m}$ then

Create $O m_{i j}^{t}$ by performing mutation on $O_{i j}^{t}$ using Eq. (44).

end if

Evaluate the objective function $F_{1}\left(O m_{i j}^{t}\right)$

Evaluate the constraint violation $F_{2}\left(O m_{i j}^{t}\right)=V\left(O m_{i j}^{t}\right)$ using Eq. (53).

Add $O_{i j}^{t}$ and $O m_{i j}^{t}$ to the total population

Sort the whole population based on $\mu_{D}^{k}$ values and select the best $N_{P}$ individuals for the next-

generation population

Update the best solution $O_{\text {best }}$ using Eq. (56). 
return the best solution $O_{\text {best }}$

\section{5- Performance Evaluation}

In this section, we first describe the settings used in the simulation. Then we compare the performance of the modified MA and the GA with the baseline and state-of-the-art methods.

\section{5-1- Experimental Setting}

We used the CloudSim tool (version 3.03) to simulate the proposed method [20]. The CloudSim was developed at the University of Melbourne, Australia. Also, parts of the analysis of results were done with SPSS software. The experiments were done on a 64-bit Intel ${ }^{\circledR}$ Core $^{\mathrm{TM}}$ i5-8269U Processor with 6 MB Cache, 4 Cores, 4.20 GHz CPU frequency, and 8 GB RAM.

To locate data centers, we have selected four cities in the United States, with different time zones and the same network infrastructure: Hampton (in Virginia state), Houston (in Texas state), Salem (in Oregon state), and San Francisco (in California state). The number of hops for each packet to reach the destination is estimated between 12 to 14 . So, the side effects of different distances in the communication network can be ignored [1]. Each data center has 130 physical machines that are configured in 5 different types according to Table 4.

Table 4- Configuration of physical machines

\begin{tabular}{|c|c|c|c|c|}
\hline Configuration Type & Core number & $\begin{array}{c}\text { Core frequency } \\
(\mathbf{G H z})\end{array}$ & Memory (MB) & Storage (GB) \\
\hline Type 1 & 2 & 1.7 & 16 & 2000 \\
\hline Type 2 & 4 & 1.7 & 32 & 6000 \\
\hline Type 3 & 8 & 1.7 & 32 & 7000 \\
\hline Type 4 & 8 & 2.4 & 64 & 7000 \\
\hline Type 5 & 8 & 2.4 & 128 & 9000 \\
\hline
\end{tabular}

\section{- Solar Energy}

We used data reported from the PVGIS-NSRDB data center on the European Commission portal [43] concerning the solar energy of the four above-mentioned cities. These data are the result of a joint venture between PVGIS-NSRDB and the National Solar Radiation Database (NREL). In this study, each PhotoVoltaic (PV) solar panel, obtained from solar cell assembly, has modules that are installed in a fixed position and do not change during the day/year. Using this data, we have the amount of solar energy per day in terms of $\mathrm{W} / \mathrm{m}^{2}$. Also, we have considered the area of each flat solar absorber plate equal to $2684 \mathrm{~m}^{2}$. This number is based on the configuration used in [1]. Therefore, by having the amount of energy produced for each piece of solar panel, we can calculate the total power of a solar power plant consisting of 2648 solar panels. The solar energy specifications are shown in Fig. 10. 


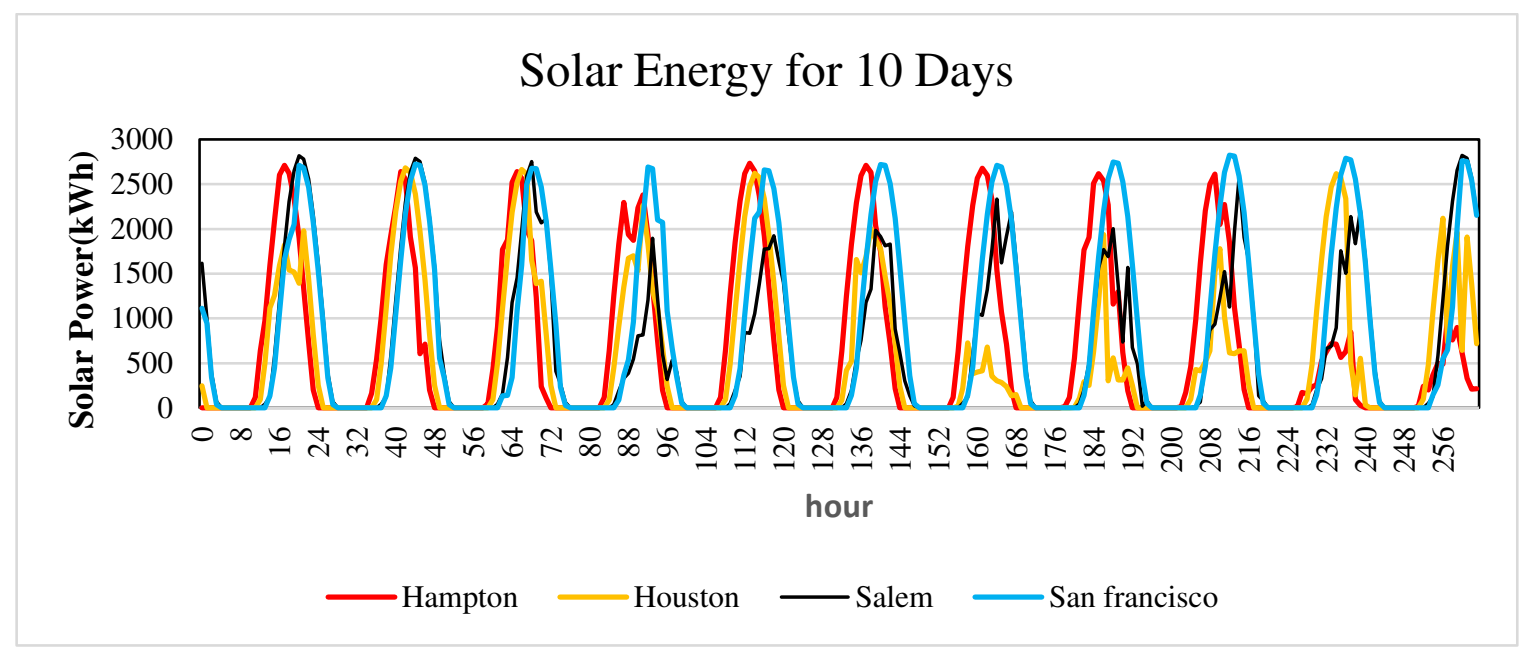

Fig. 10- Solar energy for 10 days

\section{- Outdoor Temperature}

We used data provided by the European Commission portal to obtain the ambient temperature per hour [44]. For this purpose, ambient temperature data regarding the above four cities have been collected from May 20, 2014, to May 30, 2014. Fig. 11 shows these data.

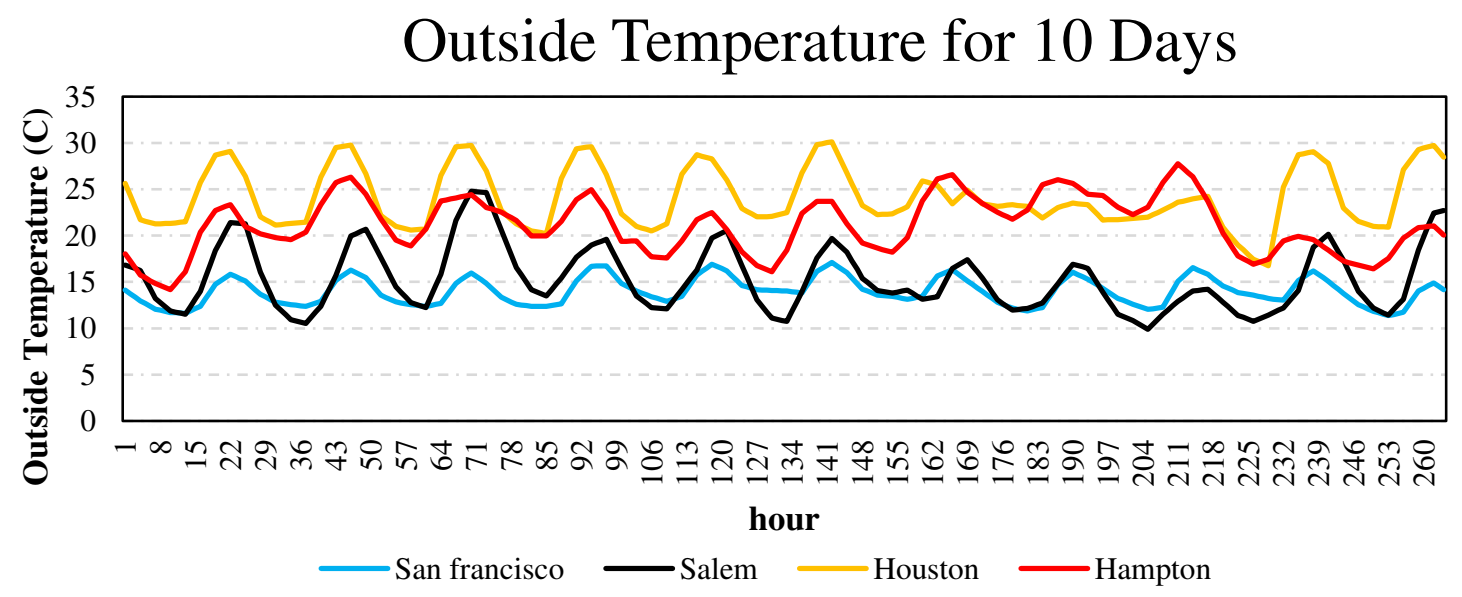

Fig. 11- Outdoor temperature for 10 days

\section{- PUE and Server Power Consumption}

We use Eq. (1), which was described earlier, to calculate the PUE. Also, it was previously stated in Eq. (2) that power has a linear relationship with CPU utilization [21]. We consider the values of $P_{h_{l}}^{\text {idle }}$ and $P_{h_{l}}^{\text {peak }}$ to be 162 and 215 watts, respectively. Therefore, Eq. (2) can be simplified as follows:

$$
P_{h_{l}}(u, t)=162+53 u_{h_{l}}(t)
$$

\section{- Carbon Dioxide Production Rate and Carbon Tax}


The rate of carbon dioxide emission in each data center is obtained from the website of the US Department of Energy [45] in TonsMWh. We have also calculated the tax on carbon dioxide emissions, which is applied in some countries [1]. The carbon dioxide tax is calculated in dollars per tonne (Dollars Tonne). These values are shown in Table 5.

\section{- Energy Cost}

The cost of consumed electricity is obtained from the website of the US Energy Information Administration (EIA) [46] concerning the four cities. The information is shown in Table 5. We estimate the cost of solar energy to be zero. In this regard, note that installation and maintenance costs are incurred only once and are not a function of the consumed solar energy. Thus, even if more solar energy is consumed, it will not impose more costs on a cloud data center.

Table 5- data center specifications $[1,45]$

\begin{tabular}{|c|c|c|c|c|}
\hline Data Center Specifications & Hampton & Houston & Salem & San Francisco \\
\hline Carbon Density Rate (Tons/MWh) & 0.43 & 0.580 & 0.138 & 0.28 \\
\hline Carbon Tax Rate (Dollars / Ton) & 22 & 24 & 48 & 11 \\
\hline Energy cost (cent/kWh) & 9.17 & 8.94 & 8.68 & 15.15 \\
\hline
\end{tabular}

\section{- Workload Data}

The workloads produced by users arrive at VMs. We select VMs' specifications based on what Amazon EC2 offers [44]. The characteristics of the applications are shown in Table 6. The workload is generated using the Lublin-Feitelson model [46], including two types: the bag of the task (in which there is no interaction with the user) and the web request. These two types of workloads have different distributions. The only difference between the two groups is holding time. Generally, web requests take longer than the bag of task requests. By modifying a few parameters from [46], we tried to create a proportional distribution of requests to generate two distinct types of user requests. According to the approach used in [1] to generate the bag of task requests, we changed the first parameter of the gamma distribution to 20.4. In this way, we generate requests with a longer holding time. We also changed the holding time distribution to Hypergamma (with an average of 73 and a variance of 165) to generate web requests.

In this research, 30 different workloads are produced, each of which is tested separately. In this way, we can make more confidence in the accuracy of the test results.

Table 6- VM types and user simulated requests (BT: Bag of Task; WR: Web request) [44]

\begin{tabular}{|l|c|c|c|c|c|c|}
\hline \multicolumn{2}{|c|}{ VM Type } & $\begin{array}{c}\text { Core } \\
\text { number }\end{array}$ & $\begin{array}{c}\text { Core } \\
\text { frequency } \\
\text { (GHz) }\end{array}$ & $\begin{array}{c}\text { Memory } \\
\text { (MB) }\end{array}$ & $\begin{array}{c}\text { Storage } \\
\text { (GB) }\end{array}$ & $\begin{array}{c}\text { Probability } \\
\text { of user type } \\
\text { request }\end{array}$ \\
\hline \multirow{2}{*}{$\begin{array}{l}\text { Standard } \\
\text { Instances }\end{array}$} & M1Small & 1 & 1 & 1740 & 160 & $0.25-\mathrm{BT}$ \\
\cline { 2 - 7 } & M1Large & 2 & 4 & 7680 & 850 & $0.12-\mathrm{WR}$ \\
& & & & & $0.25-\mathrm{BT}$ \\
\hline
\end{tabular}




\begin{tabular}{|c|c|c|c|c|c|c|}
\hline & M1XLarge & 4 & 8 & 15360 & 1690 & $0.08-\mathrm{WR}$ \\
\hline $\begin{array}{c}\text { High Memory } \\
\text { Instances }\end{array}$ & M2XLarge & 2 & 6.5 & 17510 & 420 & $0.12-\mathrm{WR}$ \\
\cline { 2 - 6 } & M22XLarge & 4 & 13 & 35020 & 850 & $0.08-\mathrm{WR}$ \\
\hline $\begin{array}{c}\text { High CPU } \\
\text { Instances }\end{array}$ & C1Medium & 2 & 5 & 1740 & 320 & $0.1-\mathrm{BT}$ \\
\hline
\end{tabular}

\section{5-2- Execution Time of Algorithms}

For the sake of fairness, we compare our proposed method with one of the state-of-the-art methods called DDMPEA. The details of this method have already been described in Section 4-3. We first use this method to solve the cost optimization sub-problem of Eq. (24) and call it CRA-DP-DDMPEA. Then, we use it to solve the joint cost and scheduling optimization of Eq. (36) and name it CRA-DPSCH-DDMPEA. So far, none of the previous studies have addressed the issue of joint optimization. Similarly, we abbreviate the solution of the cost optimization sub-problem by the MA with the symbol CRA-DP-MA. Also, solving the problem of joint cost and scheduling optimization is abbreviated with CRA-DPSCH-MA.

We now proceed to analyze the execution time of all algorithms. Roughly speaking, we want to examine the extent to which different metaheuristic methods could make a significant difference with the baseline algorithm CRA-DP.

One of the prerequisites for most statistical analyzes concerning random variables is the normality of the data. As a result, checking the normality of the data is a key point, especially when the distribution is not clear and the sample size is small. One of the criteria for data normality is the Quantile -Quantile (Q-Q) plot. There are many statistical tests such as Shapiro-Wilk, Dagostino, Anderson-Darling, and Kolmogorov-Smirnov that can be used to test the normality of the data. In this study, we use the Shapiro-Wilk and Kolmogorov-Smirnov methods to inspect the hypothesis of normality of the data $\left(\mathrm{H}_{0}\right)$. Because we assume a $95 \%$ confidence interval, the significance level is 0.05 . Assumption $\mathrm{H}_{0}$ is rejected if the value of the sig (significance level) is less than 0.05 . Table 7 shows the result of the normality test concerning the execution time of algorithms. Since the value of sig in both Shapiro-Wilk and Kolmogorov-Smirnov tests for all VMP algorithms is less than 0.05, the hypothesis $\mathrm{H}_{0}$ is rejected. In other words, the data obtained from the execution time of the algorithms are not normal. There are two ways to continue: 1) use non-parametric tests to compare the results 2) try to normalize the data so that we can use parametric methods. Note that parametric methods are more accurate than nonparametric ones. So, we choose the second way. Due to space limitations, we do not provide details on data normalization. Table 8 shows the execution time after normalization.

Table 7 - The normality test of data regarding the execution time of algorithms

\begin{tabular}{|c|c|c|c|c|c|c|}
\hline & \multicolumn{3}{|c|}{ Kolmogorov-Smirnov } & \multicolumn{3}{|c|}{ Shapiro-Wilk } \\
\hline & Statistic & $\mathrm{df}$ & Sig. & Statistic & $\mathrm{df}$ & Sig. \\
\hline FFD & .484 & 30 & .000 & .349 & 30 & .000 \\
\hline CRA-DP & .167 & 30 & .033 & .852 & 30 & .001 \\
\hline CRA-DP-GA & .297 & 30 & .000 & .569 & 30 & .000 \\
\hline
\end{tabular}




\begin{tabular}{l|l|l|l|l|l|l}
\hline CRA-DP-MA & .293 & 30 & .000 & .650 & 30 & .000 \\
\hline
\end{tabular}

a. Lilliefors Significance Correction

Table 8 - The normality test of data regarding the execution time (after normalization)

\begin{tabular}{|c|c|c|c|c|c|c|}
\hline \multicolumn{7}{|c|}{ Tests of Normality } \\
\hline & \multicolumn{3}{|c|}{ Kolmogorov-Smirnov ${ }^{\mathrm{a}}$} & \multicolumn{3}{|c|}{ Shapiro-Wilk } \\
\hline & Statistic & df & Sig. & Statistic & df & Sig. \\
\hline CRA-DP-MA & .174 & 24 & .059 & .945 & 24 & .208 \\
\hline CRA-DP-GA & .132 & 24 & $.200^{*}$ & .939 & 24 & .159 \\
\hline CRA-DP & .151 & 24 & .164 & .957 & 24 & .384 \\
\hline
\end{tabular}

The Q-Q plot for the execution time of algorithms is shown in Figs. 12, 13, and 14. As mentioned earlier, the $\mathrm{Q}-\mathrm{Q}$ plot is used to determine how close the runtime value is to the normal distribution.

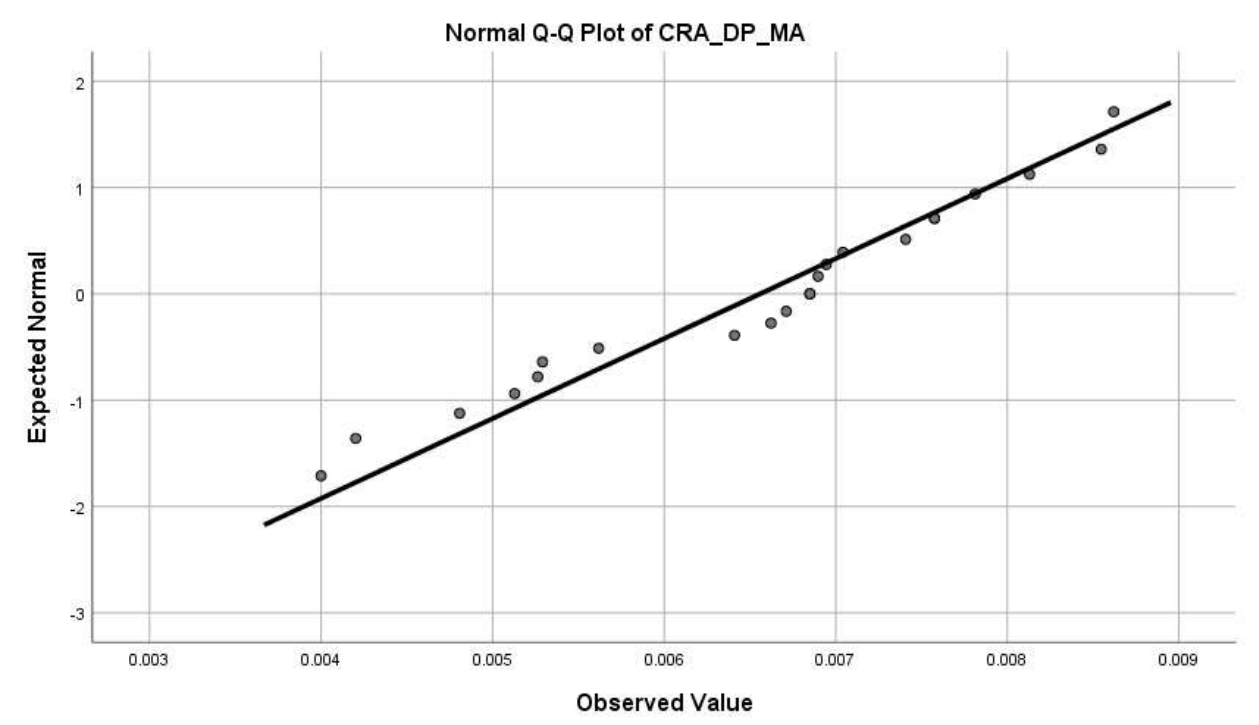

Fig. 12- Normalized Q-Q plot of CRA-DP-MA 


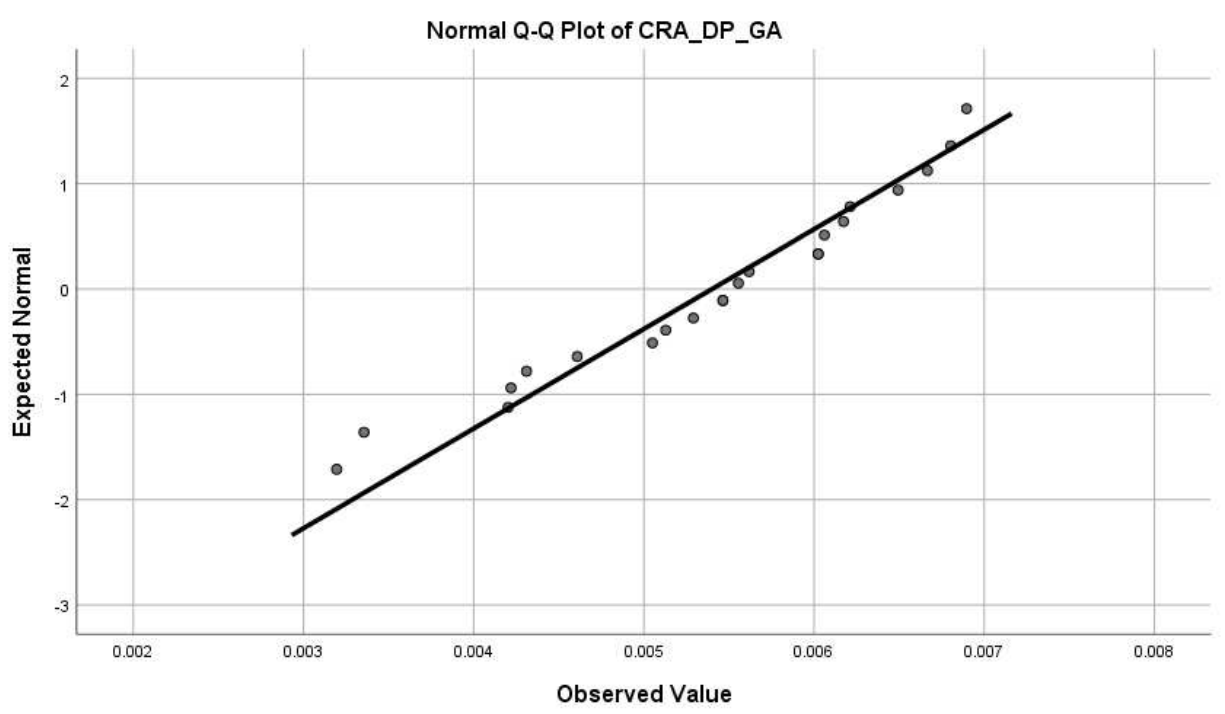

Fig. 13- Normalized Q-Q plot of CRA-DP-GA

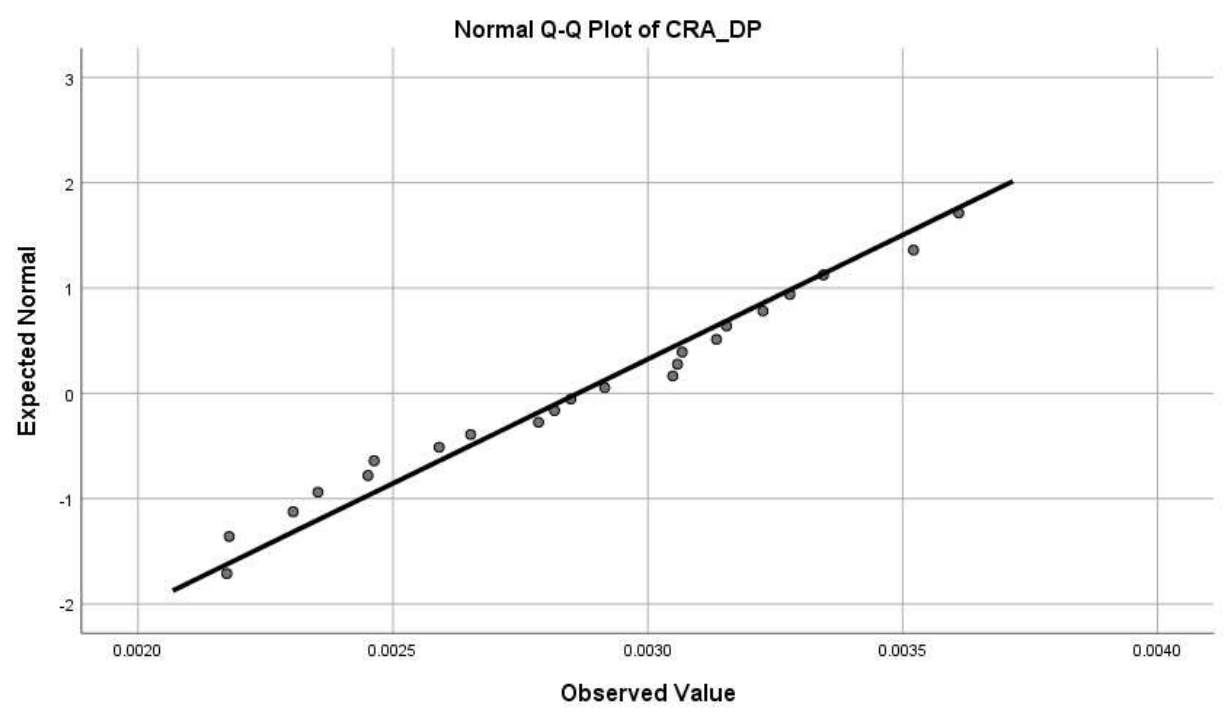

Fig. 14- Normalized Q-Q plot of CRA-DP

Now, according to the results obtained from Table 8 and the value of the Sig parameter for each algorithm, hypothesis $\mathrm{H}_{0}$ (data normality) is accepted. Therefore, to continue the analyses, we can use any parametric methods, for example, the Analysis of Variance (ANOVA) [18]. The ANOVA test hypotheses are as follows:

$\mathbf{H}_{0}$ : The average convergence time is equal in all algorithms (there is no significant difference).

$\mathbf{H}_{1}$ : The average convergence time is not equal for all algorithms (there is a significant difference).

Table 9- One-way analysis of variance (ANOVA) test for execution time

\section{ANOVA}

\begin{tabular}{ll|l|l|l} 
Sum of Squares & df & Mean Square & F & Sig. \\
\hline
\end{tabular}




\begin{tabular}{l|c|c|c|c|c}
\hline Between Groups & .000 & 2 & .000 & 104.980 & .000 \\
\hline Within Groups & .000 & 79 & .000 & & \\
\hline Total & .000 & 81 & & & \\
\hline
\end{tabular}

Table 9 shows the results of the ANOVA test. Since the value of the Sig is less than 0.05, it can be concluded that hypothesis $\mathrm{H}_{0}$ is rejected. In other words, there is a significant difference among algorithms (groups) in terms of convergence time. Apart from the normality, another prerequisite for the ANOVA test is that the variance of the data is homogeneous. This is checked by the Levin test. Due to space limitations, we do not provide details of this test. Because the variance of the data is not homogeneous, the Games-Howell post hoc test can be used for the pairwise comparison of algorithms. The results of this test are shown in Table 10. The value of Sig in all rows of the table is less than 0.05 , which indicates a significant pairwise difference among algorithms. The analysis of this table is based on the upper and lower limit values with the following rules:

1) If both the upper limit and the lower limit are positive, the difference between the mean of the two algorithms is greater than zero and the mean of the first algorithm is greater than that of the second algorithm.

2) If both the upper limit and the lower limit are negative, the difference between the mean of the two algorithms is less than zero and the mean of the first algorithm is less than that of the second algorithm.

3) If the upper limit is positive and the lower limit is negative, the difference between the means of the two algorithms is not significant and; hence, the equality of the mean of the two algorithms is not rejected.

The Mean Difference column in Table 10 shows that the means of the CRA-DP-GA and CRA-DP-MA differ by 0.0256 and 0.0376 , respectively, compared to the CRA-DP algorithm. Given the random nature of metaheuristic algorithms, this amount of discrepancy seems plausible.

Table 10- Games-Howell post hoc test

\begin{tabular}{|c|c|c|c|c|c|c|}
\hline \multicolumn{7}{|c|}{ Multiple Comparisons } \\
\hline \multicolumn{7}{|l|}{ Games-Howell } \\
\hline \multirow{2}{*}{ (I) VAR } & \multirow{2}{*}{ (J) VAR } & \multirow{2}{*}{$\begin{array}{c}\text { Mean Difference } \\
(\mathrm{I}-\mathrm{J})\end{array}$} & \multirow{2}{*}{ Std. Error } & \multirow{2}{*}{ Sig. } & \multicolumn{2}{|c|}{ 95\% Confidence Interval } \\
\hline & & & & & Lower Bound & Upper Bound \\
\hline \multirow{2}{*}{ CRA-DP } & CRA-DP-GA & $-.00256^{*}$ & .00022 & .000 & -.0031 & -.0020 \\
\hline & CRA-DP-MA & $-.00376^{*}$ & .00026 & .000 & -.0044 & -.0031 \\
\hline \multirow{2}{*}{ CRA-DP-GA } & CRA-DP & $.00256^{*}$ & .00022 & .000 & .0020 & .0031 \\
\hline & CRA-DP-MA & $-.00120^{*}$ & .00032 & .001 & -.0020 & -.0004 \\
\hline \multirow{2}{*}{ CRA-DP-MA } & CRA-DP & $.00376^{*}$ & .00026 & .000 & .0031 & .0044 \\
\hline & CRA-DP-GA & $.00120^{*}$ & .00032 & .001 & .0004 & .0020 \\
\hline
\end{tabular}

*. The mean difference is significant at the 0.05 level.

Also, the average time to find the optimal solution is shown in Fig. 15. As can be seen in the figure, there is a significant difference between the average time of the CRA-DP and other algorithms. The CRA-DP has the highest average execution time, followed by the CRA-DP-GA and CRA-DP-DDMMPEA, respectively. We expect that with the increase in the number of data centers as well as the number of physical machines, the time to find the optimal solution for the CRA-DP algorithm will increase significantly. The reason is that this 
algorithm searches most of the data centers and physical machines, which in turn makes VMP longer, while the search space in the other two metaheuristic algorithms is much more limited.

As shown in Fig. 15, joint optimization methods (CRA-DPSCH-DDMMPEA and CRA-DPSCH-MA) have a longer convergence time than methods in which only cost optimization is performed. Note that the multiobjective optimization problem presented in Eq. (36) has more constraints than the single-objective case of Eq. (24). Also, the values obtained for each function in multi-objective problems are usually less than the values obtained in single-objective cases. This is because of the tradeoff that can be made between potentially conflicting goals. This causes exploration to be slower in the search space, which in turn leads to long execution times. Although the amount of this time overhead is small, it has interesting achievements that will be explained later in Figs. 17 and 22. Also, Fig. 15 shows that the convergence time in multi-objective methods never increases by more than $4 \%$ compared to single-objective cases.

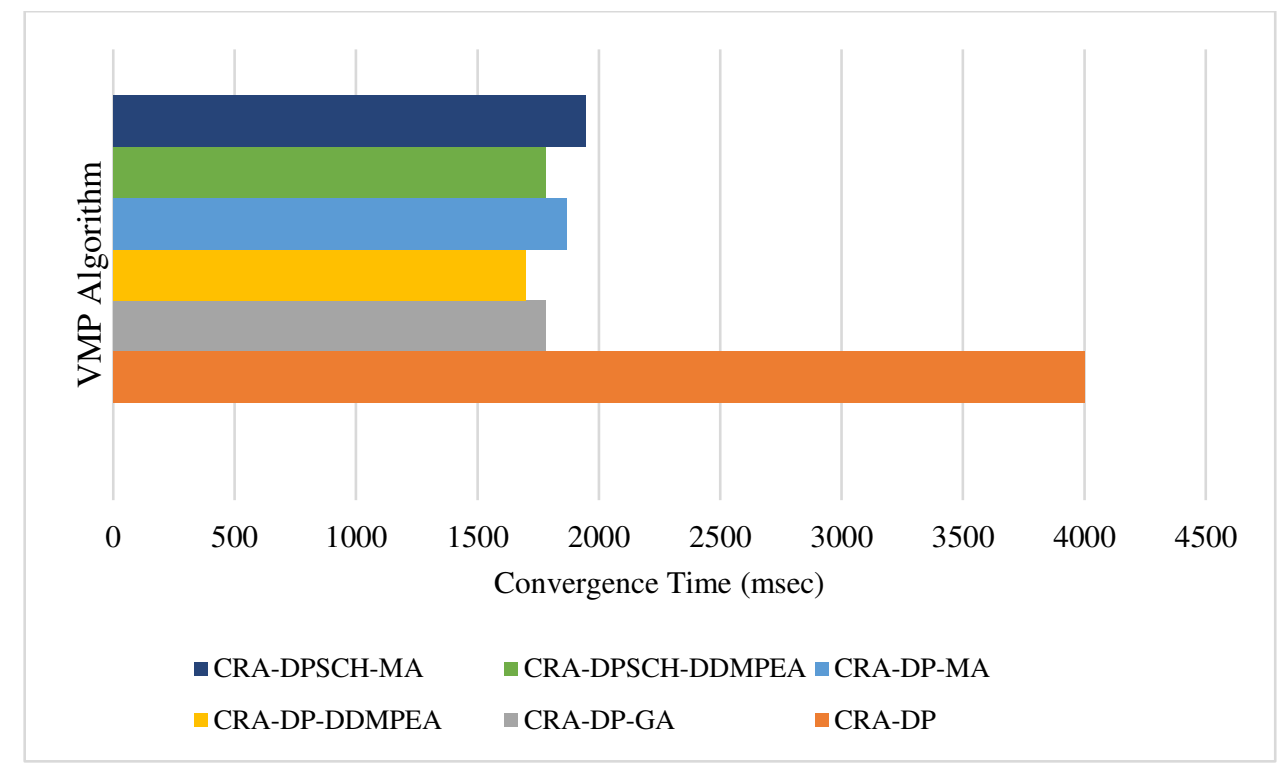

Fig. 15- Average convergence time (finding the optimal solution) for different VMP algorithms

\section{5-3- Energy Consumption}

As is shown in Fig. 16, the FFD algorithm consumes more fossil fuel for VM placement compared to other algorithms. This is because the FFD method places the VMs only based on the remaining capacity of physical servers, and no other parameter is involved in this decision. However, the CRA-DP-MA algorithm has the lowest brown energy consumption because it involves significant parameters such as PUE and data center availability. One of the most important parameters that affect the energy consumption of fossil fuels is the PUE. This parameter affects the VMP by considering both the outside temperature and the workload. The availability of renewable energy sources and the prioritization of data center power supply also have significant impacts on metaheuristic VMP algorithms. As can be seen in Fig. 16, there is no significant difference between metaheuristic algorithms. In other words, the brown energy consumption of the proposed meta-heuristic algorithms is not much higher than that of the CRA-DP algorithm. The ANOVA test, shown in Table 11, also confirms this claim. Since the value of the Sig $=0.586$ is greater than 0.05 , the hypothesis $\mathrm{H}_{0}$ is accepted. It means that the amount of brown energy consumption in the above algorithms is not significantly different. Also, 
the figure shows that the energy consumption of methods that use joint cost and scheduling optimization is slightly higher than that of single-objective methods. The general reason behind this behavior has already been stated in Fig. 15. The brown energy consumption in multi-objective methods never increases by more than $3 \%$ compared to single-objective cases.

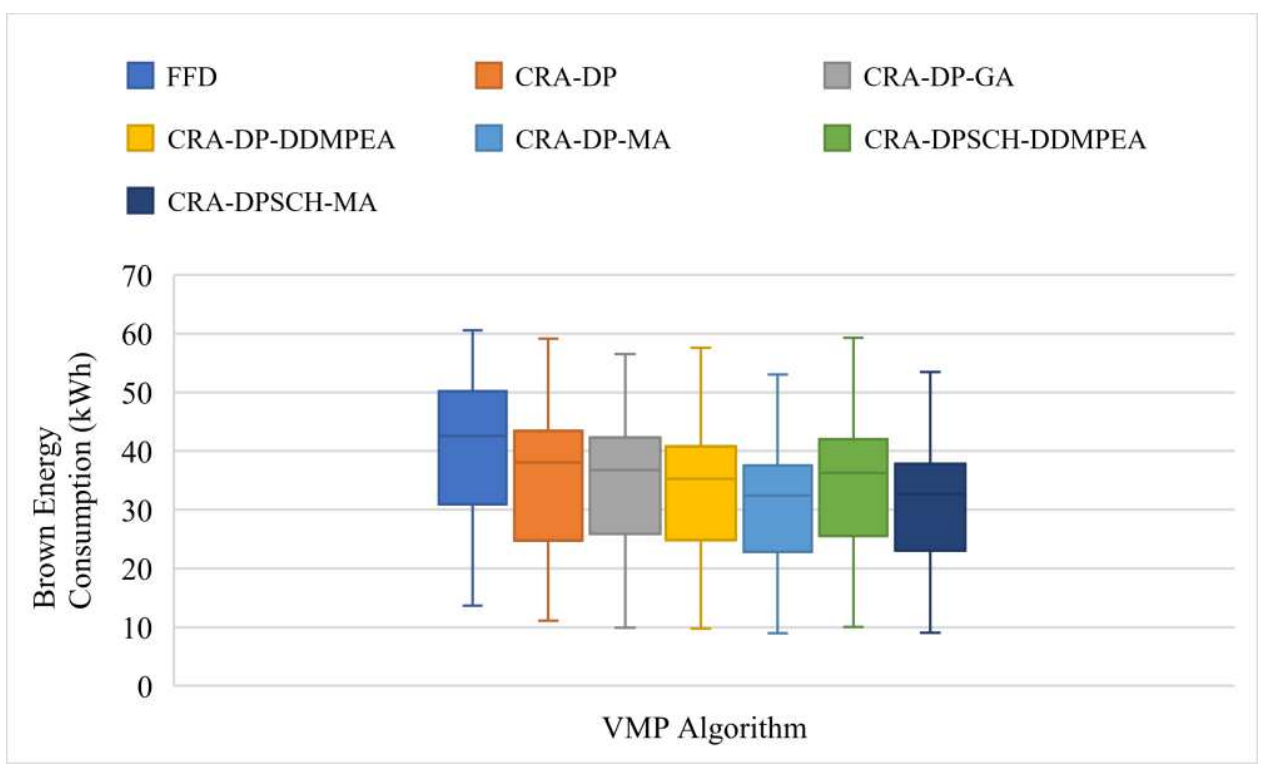

Fig. 16- Brown energy consumption (fossil fuels) for different VMP algorithms

Table 11- ANOVA test for the brown energy consumption of algorithms

\begin{tabular}{c|c|c|c|c|c}
\multicolumn{7}{c}{ ANOVA } \\
\hline & Sum of Squares & df & Mean Square & F & Sig. \\
\hline Between Groups & 186812789.689 & 2 & 93406394.844 & .538 & .586 \\
\hline Within Groups & 15105345863.467 & 87 & 173624665.097 & & \\
\hline Total & 15292158653.156 & 89 & & &
\end{tabular}

As shown in Fig. 17, the FFD algorithm has the lowest energy consumption regarding renewable sources. Given that in this algorithm, the order and availability of physical resources are the only parameters for VMP, such a result is quite expected. The interesting point in Fig. 17 is that there is very little difference between the amount of solar energy consumption in the CRA-DP-GA and the CRA-DP-MA algorithms. The reason for this behavior can be found in the neighborhood selection method in these algorithms. In the proposed neighborhood algorithm, there is a severe limitation on choosing a neighbor for a solution. We consider this limitation so that neighboring solutions can be generated with a higher chance. The fitness function in the CRA-DP-MA assigns the highest importance for solar energy. So, if we do not apply the data center constraints, we will obtain solutions with completely different fitness. Fig. 17 shows that the green energy consumption in multi-objective methods never increases by more than $4 \%$ compared to single-objective cases. Also, the ANOVA test result is 
shown in Table 12. The value of Sig. $=0.963$ indicates the acceptance of hypothesis $\mathrm{H}_{0}$. This indicates that statistically, the amount of green energy consumption by metaheuristic algorithms is not significantly different.

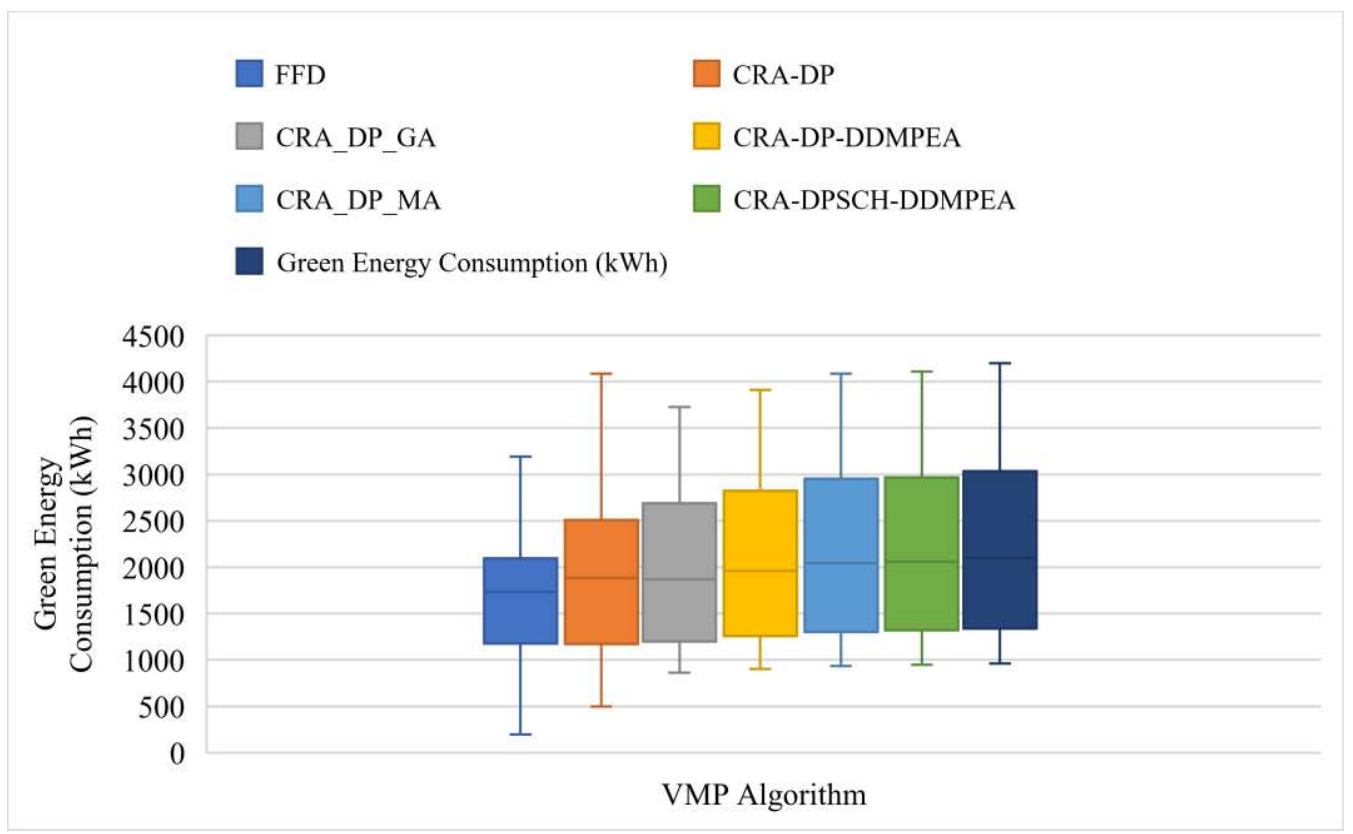

Fig. 17- Green energy consumption for different VMP algorithms

Table 12- The ANOVA test on green energy consumption

\begin{tabular}{l|c|c|c|c|c}
\multicolumn{1}{c|}{} & $\begin{array}{c}\text { Sum of } \\
\text { Squares }\end{array}$ & df & Mean Square & F & Sig. \\
\hline Between Groups & 57027.970 & 2 & 28513.985 & .038 & .963 \\
\hline Within Groups & 65193500.308 & 87 & 749350.578 & & \\
\hline Total & 65250528.278 & 89 & & &
\end{tabular}

\section{5-4- Carbon Footprint Emission}

As is shown in Fig. 18, the FFD algorithm has the highest amount of carbon dioxide production. According to the explanations provided in Section 5-3 and considering that the amount of fossil fuel consumption is directly related to carbon dioxide emissions, such good performance is quite predictable for the FFD algorithm. Similar to Fig. 16, Fig. 18 does not show a significant difference among metaheuristic methods. They do not have a significant effect on reducing the carbon footprint compared to the CRA-DP algorithm. However, they can prevent an increase in greenhouse gas emissions by reducing the convergence time. Fig. 18 shows that the carbon footprint in multi-objective methods never increases by more than $3 \%$ compared to single-objective cases. Also, the ANOVA test is shown in Table 13. The value of Sig. $=0.543$ indicates the acceptance of hypothesis $\mathrm{H}_{0}$. This indicates that the amount of produced carbon dioxide by metaheuristic algorithms is not significantly different. 


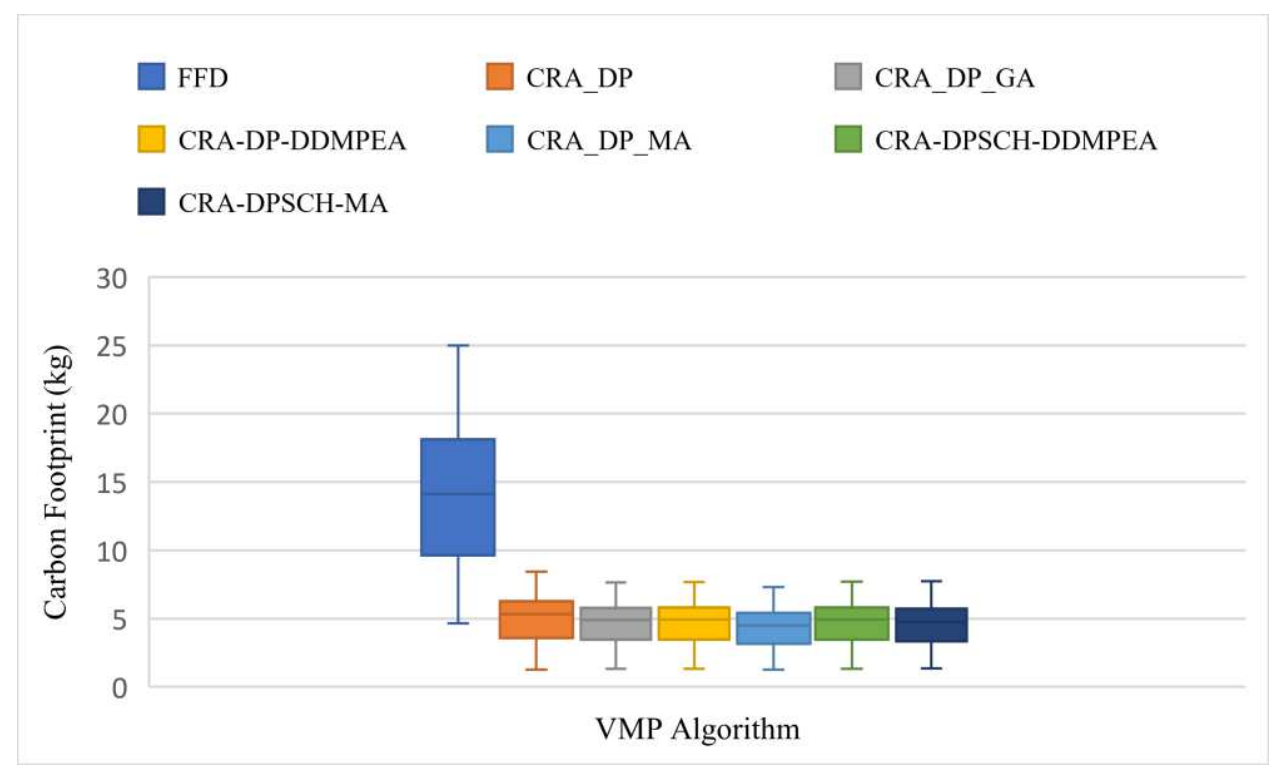

Fig. 18- Production of carbon dioxide (greenhouse gases) for different VMP algorithms

Table 13- One-way analysis of variance (ANOVA) test for carbon dioxide (greenhouse gas) emissions

\begin{tabular}{l|c|c|c|c|c}
\multicolumn{7}{c|}{} & $\begin{array}{c}\text { Sum of } \\
\text { Squares }\end{array}$ & df & Mean Square & F & Sig. \\
\hline Between Groups & 3834114.771 & 2 & 1917057.386 & .614 & .543 \\
\hline Within Groups & 271418811.035 & 87 & 3119756.449 & & \\
\hline Total & 275252925.806 & 89 & & &
\end{tabular}

\section{5-5- Cost}

According to Eqs. (3)-(4), the energy cost is the sum of the green energy (renewable) cost and the brown energy (fossil fuels) cost. As mentioned earlier, since the cost of renewable energy is low in the long-term run, we set it at zero. As a result, the energy cost is only a function of the amount of consumed fossil fuel. In Fig. 19, the CRA-DP-MA, with a negligible difference, consumes less energy than the CRA-DP-GA, CRA-DP-DDMPEA, and the CRA-DP algorithms. The reason for this behavior was previously described in Fig. 16. 


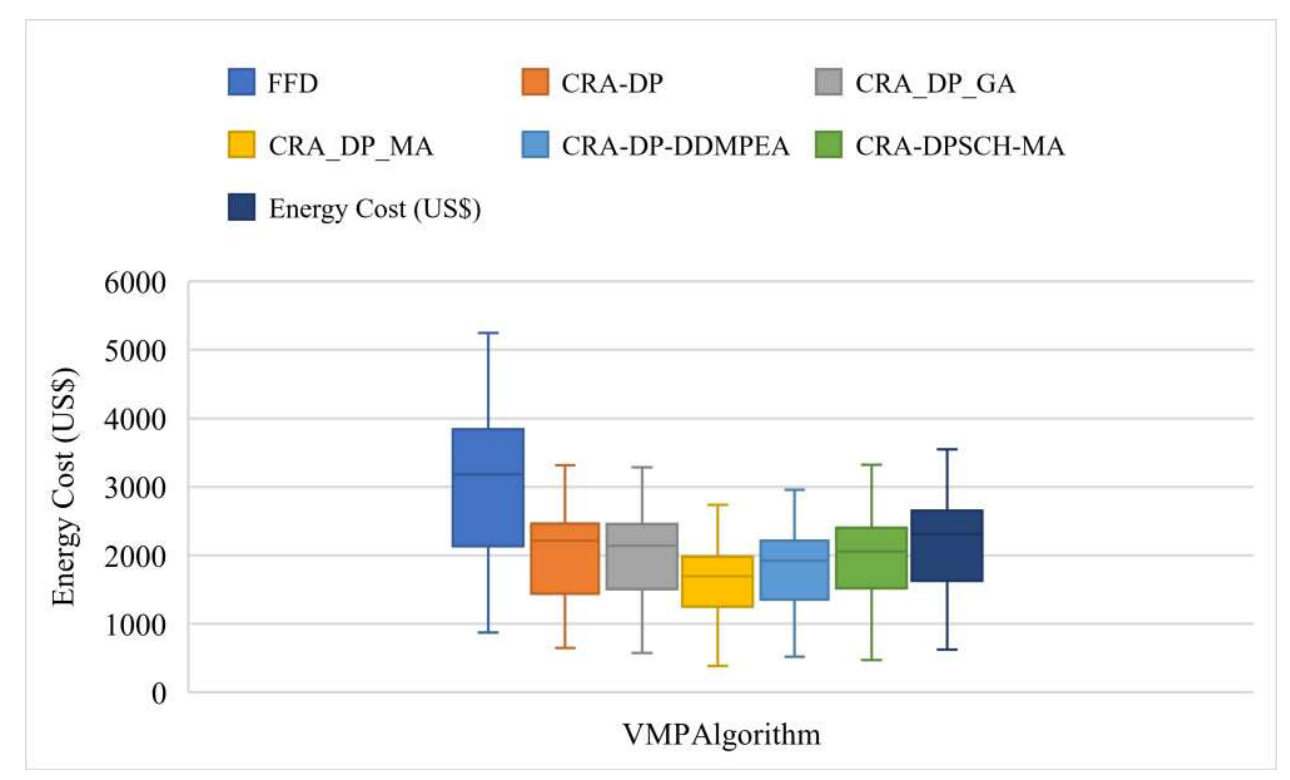

Fig. 19- Energy cost for different VMP algorithms

Figs. 20 and 21 show the carbon cost and the total cost, respectively. According to Eq. (18), the carbon cost is a function of the rate of produced carbon dioxide and the associated tax. As can be seen in the figure, the amount of carbon produced by metaheuristic methods is never significantly worse than that of the baseline method, $C R A-D P$. Fig. 20 shows that the cost of carbon in multi-objective methods never increases by more than $4 \%$ compared to single-objective cases. Similarly, Fig. 21 shows that the total cost in multi-objective methods never increases by more than $5 \%$ compared to single-objective cases.

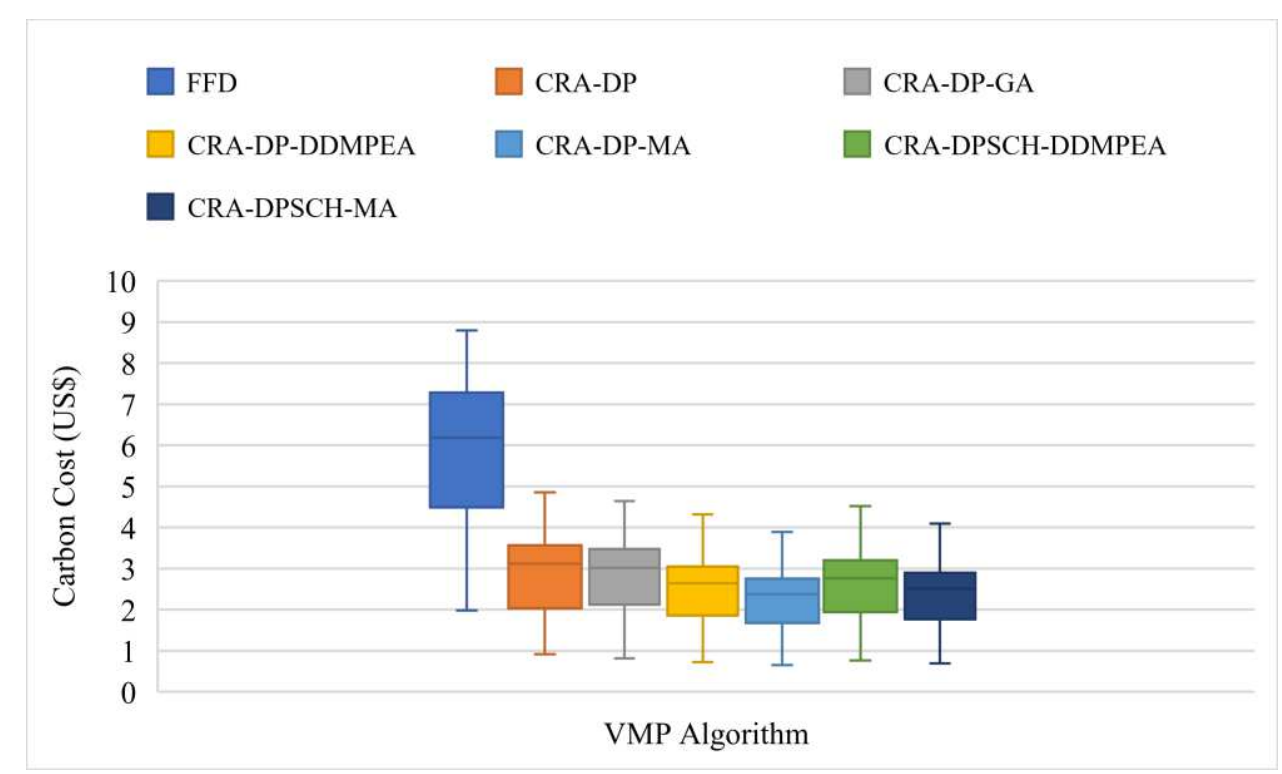

Fig. 20- The cost of produced carbon for different VMP algorithms 


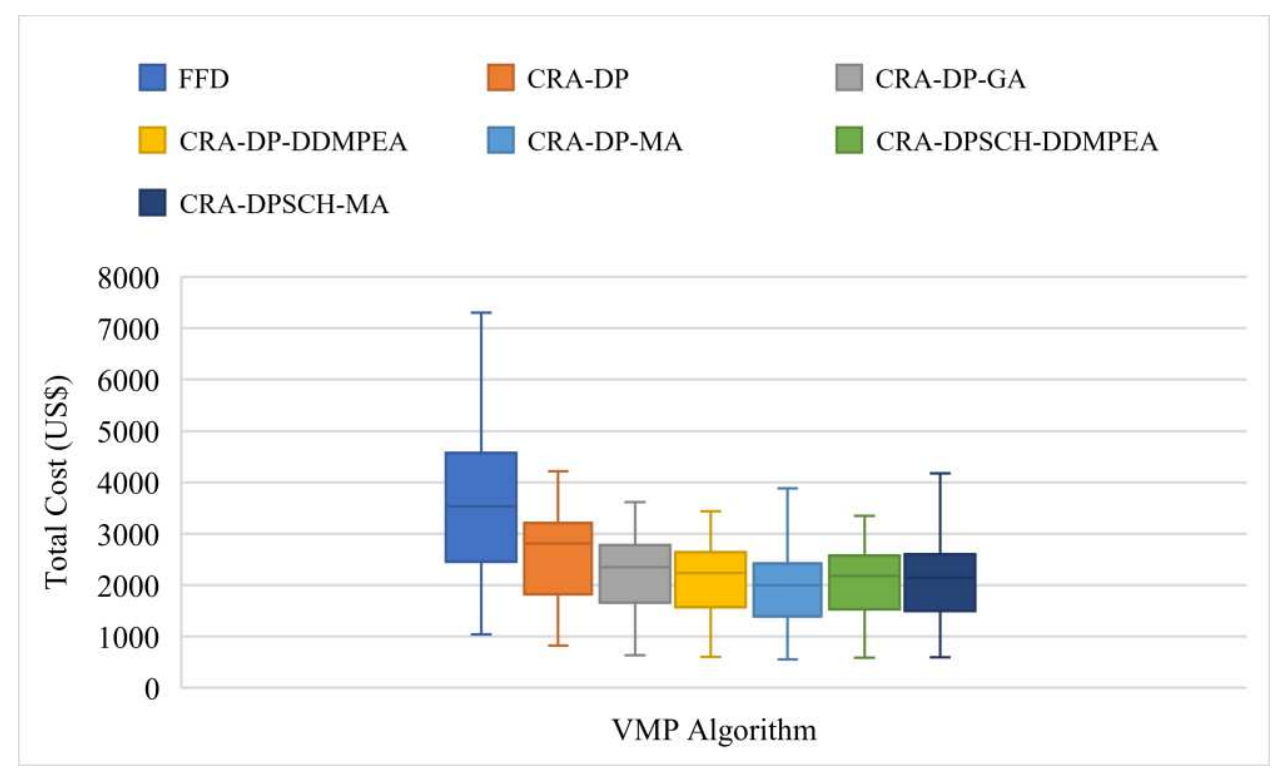

Fig. 21- Total cost for different VMP algorithms

\section{5-6- SLA Violations}

By the SLA violation we mean the number of VM requests rejected due to the lack of physical resources in the data center. We run a stress test on the system and changed the number of VM requests from 1,000 to 1,700. Fig. 22 shows the percentage of SLA violations for different algorithms. As can be seen in the figure, the more traffic intensity the system exerts, the lower is the SLA violations. This, in turn, degrades the QoE of users. However, the amount of degradation in methods that use joint cost and scheduling optimization is far less than single-objective ones. The results show that in heavy traffic, the proposed method can reduce SLA violations by almost twice. This confirms exactly our initial claim in this study about the need to pay attention to the scheduling of tasks. 


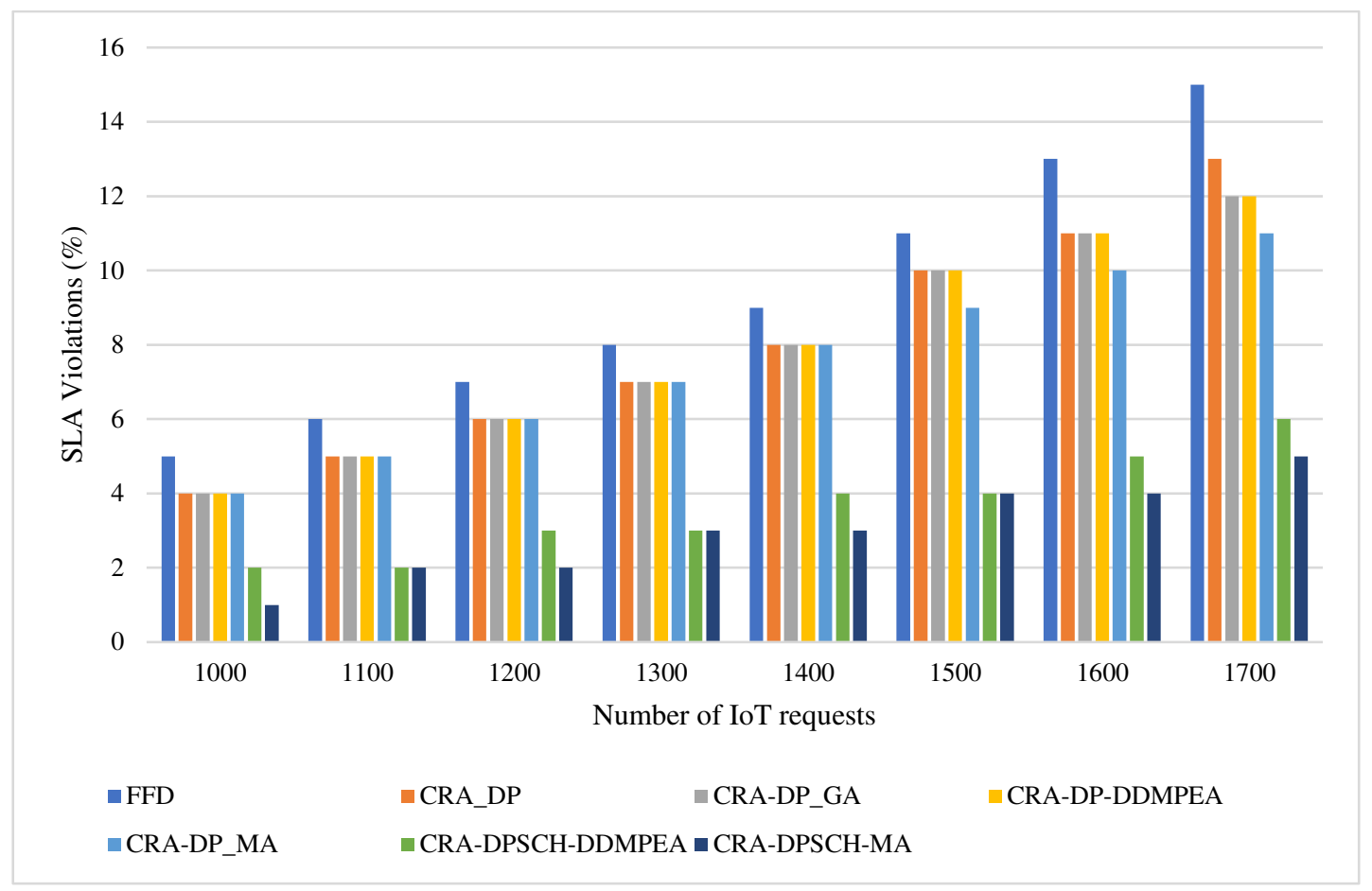

Fig. 22- Percentage of SLA violations for different number of IoT tasks

\section{6- Conclusion and Future Trends}

In this paper, we targeted one of the most practical issues of cloud computing, namely carbon-efficient VM placement. To meet real-world requirements, we incorporated the delay-sensitive nature of IoT requests into modeling. After formulating each of the two sub-problems, we provided a joint energy cost and scheduling optimization for the problem. Since the problem is NP-hard, we solved it on a large scale using the modified Memetic Algorithm (MA). Then, we evaluated the performance of the proposed method against the baseline method such as the Genetic Algorithm (GA) as well as state-of-the-art methods.

Solar energy was also considered as one of the energy supply sources to reduce energy and carbon consumption costs. Our solution not only minimizes the energy consumption of cloud service providers but also considers the users' QoE and SLA requirements. The simulation results revealed the superiority of the proposed approaches, in terms of significant criteria such as VM allocation time, energy cost, energy consumption, Power Usage Effectiveness (PUE), and SLA violation. The results showed that proposed metaheuristic algorithms can significantly decrease the convergence time compared to GA-based and the heuristic method and at the same time, do not cause a significant increase in energy consumption and cost.

For the sake of fairness, a comparison was made between multi-objective and single-objective optimization modes. As is common in optimization theory, we expect the value of the functions obtained in multi-objective optimization to be less than those of a single-objective case. However, the amount of degradation in various criteria was never more than 5\%, which is quite promising. Also, the results showed that in heavy traffic, the proposed method can reduce SLA violations by almost twice. When most tasks do not have a firm deadline, the competitive advantage of the proposed method over the base method may not be as significant. However, in the worst case, it will be as efficient as the base method. 
There are several possible lines of research for the future. To find a neighborhood in the MA, both the outside temperature and renewable energy have a significant impact on data center utilization. In this study, we assumed that hosts in the same geographical locations are more likely to have similar temperatures and solar energy. As one of the future works, the effect of these two parameters can be examined regardless of the geographical location. We also ignored the effect of communication network components. One of the future research could be to consider the impact of these components on energy consumption and the VMP cost. Machine learning methods, especially reinforcement learning, can also be used to predict future carbon-efficient placements.

\section{Compliance with ethical standards}

Conflict of interest On behalf of all authors, the corresponding author states that there is no conflict of interest.

Funding The authors did not receive support from any organization for the submitted work.

Data Availability The datasets generated during and analyzed during the current study are available in the [MENDELEY] repository, [http://data.mendeley.com/datasets/2g7dy8bnfj/1].

\section{References}

[1] Khosravi, A., Andrew, L.L. and Buyya, R., 2017. Dynamic VM placement method for minimizing energy and carbon cost in geographically distributed cloud data centers. IEEE Transactions on Sustainable Computing, 2(2), pp.183196.

[2] Ahvar, E., Orgerie, A.C. and Lebre, A., 2019. Estimating energy consumption of cloud, fog and edge computing infrastructures. IEEE Transactions on Sustainable Computing.

[3] Thiam, C. and Thiam, F., 2019. Optimizing Electrical energy Consumption in Cloud Data Center.

[4] Xu, M. and Buyya, R., 2020. Managing renewable energy and carbon footprint in multi-cloud computing environments. Journal of Parallel and Distributed Computing, 135, pp.191-202.

[5] López, J., Kushik, N. and Zeghlache, D., 2019. Virtual machine placement quality estimation in cloud infrastructures using integer linear programming. Software Quality Journal, 27(2), pp.731-755.

[6] Parvizi, E. and Rezvani, M.H., 2020. Utilization-aware energy-efficient virtual machine placement in cloud networks using NSGA-III meta-heuristic approach. Cluster Computing, pp.1-23.

[7] Aboutorabi, S.J.S. and Rezvani, M.H., 2020. An Optimized Meta-heuristic Bees Algorithm for Players' Frame Rate Allocation Problem in Cloud Gaming Environments. The Computer Games Journal, 9(3), pp.281-304.

[8] Tavakoli-Someh, S. and Rezvani, M.H., 2019. Multi-objective virtual network function placement using NSGA-II meta-heuristic approach. The Journal of Supercomputing, 75(10), pp.6451-6487.

[9] Mohammadi, A. and Rezvani, M.H., 2019. A novel optimized approach for resource reservation in cloud computing using producer-consumer theory of microeconomics. The Journal of Supercomputing, 75(11), pp.7391-7425.

[10] Laganà, D., Mastroianni, C., Meo, M. and Renga, D., 2018. Reducing the operational cost of cloud data centers through renewable energy. Algorithms, 11(10), p.145.

[11] Iwendi, C., Maddikunta, P.K.R., Gadekallu, T.R., Lakshmanna, K., Bashir, A.K. and Piran, M.J., 2021. A metaheuristic optimization approach for energy efficiency in the IoT networks. Software: Practice and Experience, 51(12), pp.2558-2571. 
[12] RM, S.P., Bhattacharya, S., Maddikunta, P.K.R., Somayaji, S.R.K., Lakshmanna, K., Kaluri, R., Hussien, A. and Gadekallu, T.R., 2020. Load balancing of energy cloud using wind driven and firefly algorithms in internet of everything. Journal of parallel and distributed computing, 142, pp.16-26.

[13] Zhang, X., Wu, T., Chen, M., Wei, T., Zhou, J., Hu, S. and Buyya, R., 2019. Energy-aware virtual machine allocation for cloud with resource reservation. Journal of Systems and Software, 147, pp.147-161.

[14] Rashida, S.Y., Sabaei, M., Ebadzadeh, M.M. and Rahmani, A.M., 2019. A memetic grouping genetic algorithm for cost efficient VM placement in multi-cloud environment. Cluster Computing, pp.1-40.

[15] Zhao, H., Wang, J., Liu, F., Wang, Q., Zhang, W. and Zheng, Q., 2018. Power-aware and performance-guaranteed virtual machine placement in the cloud. IEEE Transactions on Parallel and Distributed Systems, 29(6), pp.13851400.

[16] Khoobkar, M.H., Dehghan Takht Fooladi, M., Rezvani, M.H., Gilanian Sadeghi,M.M.,, 2022, BLMDP: Partial Offloading with Stable Equilibrium in Fog-cloud Environments using Replicator Dynamics of Evolutionary Game Theory, Cluster Computing, to be appear.

[17] Esfandiari, S. and Rezvani, M.H., 2020. An optimized content delivery approach based on demand-supply theory in disruption-tolerant networks. Telecommunication Systems, pp.1-25.

[18] Babazadeh Nanehkaran, A. and Rezvani, M.H., 2021. An Incentive-Compatible Routing Protocol for DelayTolerant Networks Using Second-Price Sealed-Bid Auction Mechanism. Wireless Personal Communications, 121(3), pp.1547-1576.

[19] Karuppiah, S.V. and Gurunathan, G., 2021. Secured storage and disease prediction of E-health data in cloud. Journal of Ambient Intelligence and Humanized Computing, 12(6), pp.6295-6306.

[20] Rodrigo N. Calheiros, Rajiv Ranjan, Anton Beloglazov, César A. F. De Rose, Rajkumar Buyya, "CloudSim: a toolkit for modeling and simulation of cloud computing environments and evaluation of resource provisioning algorithms", software practice and experience journal, Volume : 41, Issue1, pp.23 - 50, 2011.

[21] Gao, Y., Guan, H., Qi, Z., Hou, Y. and Liu, L., 2013. A multi-objective ant colony system algorithm for virtual machine placement in cloud computing. Journal of computer and system sciences, 79(8), pp.1230-1242.

[22] Misra, S.K. and Kuila, P., 2022. Energy-Efficient Task Scheduling Using Quantum-Inspired Genetic Algorithm for Cloud Data Center. In Advanced Computational Paradigms and Hybrid Intelligent Computing (pp. 467-477). Springer, Singapore.

[23] Keshavarznejad, M. Rezvani, M.H., Adabi, S. 2020. delay-aware optimization of energy consumption for task offloading in fog environments using metaheuristic algorithms. Cluster Computing, 24, pp. 1825-1853.

[24] Maddikunta, P.K.R., Gadekallu, T.R., Kaluri, R., Srivastava, G., Parizi, R.M. and Khan, M.S., 2020. Green communication in IoT networks using a hybrid optimization algorithm. Computer Communications, 159, pp.97-107.

[25] Chauhan, S., Singh, M. and Aggarwal, A.K., 2021. Cluster Head Selection in Heterogeneous Wireless Sensor Network Using a New Evolutionary Algorithm. Wireless Personal Communications, pp.1-32.

[26] Vashishtha, G. and Kumar, R., 2022. An amended grey wolf optimization with mutation strategy to diagnose bucket defects in Pelton wheel. Measurement, 187, p.110272.

[27] Chauhan, S., Singh, M. and Aggarwal, A.K., 2021. Design of a Two-Channel Quadrature Mirror Filter Bank Through a Diversity-Driven Multi-Parent Evolutionary Algorithm. Circuits, Systems, and Signal Processing, 40(7), pp.3374-3394.

[28] Vashishtha, G. and Kumar, R., 2021. Autocorrelation energy and aquila optimizer for MED filtering of sound signal to detect bearing defect in Francis turbine. Measurement Science and Technology, 33(1), p.015006.

[29] Vashishtha, G., Chauhan, S., Singh, M. and Kumar, R., 2021. Bearing defect identification by swarm decomposition considering permutation entropy measure and opposition-based slime mould algorithm. Measurement, 178, p.109389. 
[30] Vashishtha, G. and Kumar, R., 2021. Pelton Wheel Bucket Fault Diagnosis Using Improved Shannon Entropy and Expectation Maximization Principal Component Analysis. Journal of Vibration Engineering \& Technologies, pp.115.

[31] Brady, G.A., Kapur, N., Summers, J.L. and Thompson, H.M., 2013. A case study and critical assessment in calculating power usage effectiveness for a data centre. Energy Conversion and Management, 76, pp.155-161.

[32] Fawaz, A.H., Mohammed, A.F.Y., Laku, L.I.Y. and Alanazi, R., 2019, February. PUE or GPUE: A Carbon-Aware Metric for Data Centers. In 2019 21st International Conference on Advanced Communication Technology (ICACT) (pp. 38-41). IEEE.

[33] Rasmussen, N., American Power Conversion Denmark ApS, 2009. Electrical efficiency measurement for data centers. U.S. Patent Application 12/260,448.

[34] Burke, E.K., Hyde, M.R. and Kendall, G., 2006. Evolving bin packing heuristics with genetic programming. In Parallel Problem Solving from Nature-PPSN IX (pp. 860-869). Springer, Berlin, Heidelberg.

[35] Chekuri, C., 1998. Approximation algorithms for scheduling problems. stanford university.

[36] Jafari, V. and Rezvani, M.H., 2021. Joint optimization of energy consumption and time delay in IoT-fog-cloud computing environments using NSGA-II metaheuristic algorithm. Journal of Ambient Intelligence and Humanized Computing, pp.1-24.

[37] Russell, S. and Norvig, P., 2002. Artificial intelligence: a modern approach.

[38] Chauhan, S., Singh, M. and Aggarwal, A.K., 2021. Diversity driven multi-parent evolutionary algorithm with adaptive non-uniform mutation. Journal of Experimental \& Theoretical Artificial Intelligence, 33(5), pp.775-806.

[39] Chauhan, S. and Vashishtha, G., 2021, June. Mutation-based Arithmetic Optimization Algorithm for Global Optimization. In 2021 International Conference on Intelligent Technologies (CONIT) (pp. 1-6). IEEE.

[40] Besharati, R., Rezvani, M.H. and Sadeghi, M.M.G., 2021. An Incentive-compatible offloading mechanism in fogcloud environments using second-price sealed-bid auction. Journal of Grid Computing, 19(3), pp.1-29.

[41] Chauhan, S., Vashishtha, G. and Kumar, A., 2021. A symbiosis of arithmetic optimizer with slime mould algorithm for improving global optimization and conventional design problem. The Journal of Supercomputing, pp.1-41.

[42] Naghdehforoushha, M., Dehghan Takht Fooladi, M., Rezvani, M.H., Gilanian Sadeghi,M.M.,, 2022, BLMDP: A New Bi-level Markov Decision Process Approach to Joint Bidding and Task-Scheduling in Cloud Spot Market, Turk J Elec Eng \& Comp Sci, DOI: 10.3906/elk-2108-89

[43] Commission, E. 2014; Available from: European Commission.

[44] Amazon. Amazon EC2 Instance Types. Available from: https://aws.amazon.com/ec2/instance-types/.

[45] Administration, U.S.E.I. State Electricity Profiles. 2014; Available from: https://www.eia.gov/electricity/state/archive/2014/.

[46] workload, p. parallel workload Archive. Available from: https://www.cs.huji.ac.il/labs/parallel/workload/ 